Images Re-vues

re-VuES

Histoire, anthropologie et théorie de l'art

$8 \mid 2011$

Figurer les invisibles

\title{
Les invisibles des futuristes
}

\section{Costanza Bertolotti}

\section{(2) OpenEdition}

\section{Journals}

Édition électronique

URL : http://journals.openedition.org/imagesrevues/475

DOI : 10.4000/imagesrevues.475

ISSN : 1778-3801

\section{Éditeur :}

Centre d'Histoire et Théorie des Arts, Groupe d'Anthropologie Historique de l'Occident Médiéval,

Laboratoire d'Anthropologie Sociale, UMR 8210 Anthropologie et Histoire des Mondes Antiques

\section{Référence électronique}

Costanza Bertolotti, «Les invisibles des futuristes », Images Re-vues [En ligne], 8 | 2011, mis en ligne le 20 avril 2011, consulté le 02 mars 2021. URL : http://journals.openedition.org/imagesrevues/475 ;

DOI : https://doi.org/10.4000/imagesrevues.475

\section{(c) (7) (9)}

Images Re-vues est mise à disposition selon les termes de la Licence Creative Commons Attribution Pas d'Utilisation Commerciale 4.0 International. 


\title{
LES INVISIBLES DES FUTURISTES
}

\author{
Costanza Bertolotti
}

Dès 1910 les peintres futuristes ont déclaré que leur art pouvait rendre perceptible, grâce à un langage plus suggestif que mimétique, l'existence de forces invisibles et insaisissables à l'oeil nu. Pour comprendre de quelles forces il s'agit et d'où ce propos jaillit, il ne suffit pas se rapporter aux intérêts des futuristes pour l'occultisme, mais il convient de considérer également le rôle joué dans le développement de la peinture futuriste par les expériences sociales de la modernité, parmi lesquelles la foule, thème de fameux tableaux futuristes, est l'une des plus frappantes. En dépit de l'image traditionnelle d'une avant-garde consacrée à l'idole de la vitesse, l'analyse révèle un visage inédit du futurisme, marqué par la dialectique entre modernité et survivances magiques.

Les peintres futuristes ont souvent déclaré vouloir rendre visible ce qui jusqu'alors n'avait jamais été perçu ou avait toujours été considéré comme immatériel et intangible. L'article se propose d'expliquer de quelle nature étaient les invisibles qu'ils entendaient figurer, de quelles expériences sociales et suggestions intellectuelles naissait leur croyance dans l'existence de forces occultes et quelle relation s'instaura entre ces opinions et l'expérimentation artistique qui amena Boccioni et d'autres peintres futuristes à développer un nouveau langage pictural. Nous parcourrons ce trajet à l'envers, en partant de l'analyse de la notion de primitivisme futuriste, qui, comme on le verra, n'implique pas tant l'emprunt d'éléments figuratifs que le refus de la notion de représentation mimétique propre à la tradition artistique moderne et la récupération d'un rapport entre art et réalité fondé sur la suggestion que les futuristes considéraient propre à l'art archaïque et tribal. Que voulaient-ils suggérer dans leurs tableaux? C'est la question que nous nous poserons dans le deuxième paragraphe. Nous chercherons ainsi à démontrer que l'intérêt des futuristes pour l'occultisme ne suffit pas pour comprendre leur projet consistant à rendre visible l'invisible : leur perception des forces occultes qui gouvernaient le monde ne naissait pas seulement de suggestions purement intellectuelles, mais aussi d'expériences sociales de la modernité, parmi lesquelles la plus puissante qui s'imposa à eux fut celle de la foule. Si la foule joua un rôle décisif dans l'élaboration artistique de Boccioni et de Carrà, l'interprétation que les artistes (aussi bien que les psychologues) donnèrent de ce phénomène implique précisément de supposer que des liens invisibles entraînaient les individus. À la lumière de l'expérience de la foule, il est alors possible de mieux comprendre également la dialectique, apparemment contradictoire, qui s'instaura dans le futurisme entre scientisme et survivances magiques. On retrouve à cette même époque dans certains courants de l'anarchisme individualiste, avec lesquels les futuristes avaient d'étroites relations, un pareil mélange de croyances occultes et de souffle positiviste: ce n'est pas un hasard si le thème de la foule revêt dans la pensée anarchiste autant d'importance que dans la réflexion des peintres futuristes. 
1.

Lorsque l'on évoque les relations entre avant-gardes historiques et primitivisme, on attribue souvent un rôle marginal au futurisme. En 1914, Carrà liquidait violemment « toutes ces formes variées de faux primitivisme qui, à cause d'un phénomène d'aveuglement collectif, dominent l'Europe artistique $»^{1}$. Cette sentence a contribué à interdire toute tentative d'étudier l'intérêt que les futuristes ont pu avoir pour des expressions artistiques d'époques archaïques ou de cultures non occidentales. Il nous semble au contraire opportun d'examiner plus profondément la question.

Car, si en juin 1914 Carrà se prononçait contre «l'idée tout à fait fausse que l'on peut se créer une virginité de façon artificielle et une sensibilité moderne en s'exilant dans le lointain centre de l'Afrique $»^{2}$, en mars de l'année précédente, Boccioni (dans un article paru dans Lacerba) avait exprimé sa vive appréciation pour la vogue primitiviste qui avait inséré « les ouvres d'art des sauvages [...] dans le processus de rénovation moderne ». Il écrivait : «le voyage de Gauguin à Tahiti, l'apparition des idoles et fétiches du Centre-Afrique dans les ateliers de nos amis de Montmartre, sont une fatalité historique dans le domaine de la sensibilité européenne, comme l'est l'invasion d'une race barbare dans l'organisation d'un peuple décadent $»^{3}$. Du reste, dans la conclusion du Manifeste Technique de la Peinture Futuriste du 11 avril 1910, les futuristes s'étaient déjà autoproclamés «les primitifs d'une nouvelle sensibilité totalement renouvelée $»^{4}$. Un an après, dans la préface au catalogue de l'exposition parisienne, Boccioni mettait au point le concept de «primitivisme futuriste $»^{5}$.

D’autres indices encore nous poussent à considérer les affirmations lapidaires de Carrà avec circonspection. Récemment, Alessandro Del Puppo a émis l'hypothèse selon laquelle Carrà se serait aussi inspiré des masques Fang dans son travail «antigrazioso » des années 1915-1916 ${ }^{6}$. Massimo Carrà a reconnu le premier qu’à côté de Giotto, Paolo Uccello et Rousseau, «l'art nègre et primitif » a pu constituer un point de référence pour son père lors de son passage du futurisme à la période métaphysique ${ }^{7}$. On pourrait supposer que, juste au moment où l'artiste a entrepris cette réflexion qui allait ensuite le pousser à abandonner le mouvement futuriste (dans le contexte du retour à l'ordre qui s'était imposé sur la scène artistique européenne d'après guerre), l'art ainsi-dit primitif, découvert au cours des années précédentes sans doute à Paris, soit apparu aux yeux de Carrà sous une lumière nouvelle. Pour Maria Grazia Messina, «l'exemple du Modigliani sculpteur » (chez qui on trouvait « une variété de suggestions primitives ») aurait marqué profondément Carrà qui rencontra l'artiste livournais en 1912 à Paris ${ }^{8}$. Il faut noter aussi que Carrà avait déjà connu les ouvres des populations de l'Afrique et de l'Océanie auparavant. En effet, en 1900, lors de son premier séjour à Paris, Carrà avait visité le musée du Trocadéro, qu'il avait d'ailleurs trouvé

\footnotetext{
${ }^{1}$ C. Carrà, «Vita moderna e arte popolare », Lacerba $1^{\mathrm{er}}$ juin 1914, et in Id., Tutti gli Scritti, (a cura di M. Carrà), Milan, Feltrinelli, 1978, p. 34 (les citations des écrits de Carrà, que j’ai traduites moi-même, sont tirées de cette édition et quand ce n'est pas le cas, je le spécifie).

${ }^{2}$ Ibid..

${ }^{3}$ U. Boccioni, «Fondamento plastico della scultura e pittura futuriste », Lacerba 15 mars 1913, et in id., Gli Scritti Editi e Inediti, (a cura di Z. Birolli), p. 40. (les citations des écrits de Boccioni, que j'ai traduites moi-même, sont tirées de cette édition et quand ce n’est pas le cas, je le spécifie).

4 «Pittura futurista. Manifesto tecnico », in ibid., p. 11. Voir le «Manifeste des peintres futuristes », republié dans G. Lista, Futurisme. Documents, manifestes, proclamations, Lausanne, L'Age d'Homme, 1973, p. 165. (mais la traduction française ne correspond pas parfaitement à la version italienne).

${ }_{5}^{5}$ «Les exposants au public », in Les Peintres Futuristes Italiens (exposition 5-24 février 1912, Galerie BernheimJeune et Compagnie), Paris, Bernheim-Jeune et Compagnie, 1912, p. 7.

${ }^{6}$ A. Del Puppo, Primitivismo, dossier monographique de la revue Art e Dossier, Florence, Giunti, 2003, p. 25.

${ }^{7}$ La notation de M. Carrà est formulée dans C. Carrà, Tutti gli Scritti, op.cit., p. 59.

${ }^{8}$ Voir M. G. Messina, Le Muse d'Oltremare. Esotismo e primitivismo dell'arte contemporanea, Turin, Einaudi, 1993, p. 199.
} 
«plutôt sordide et sans intérêt » ${ }^{9}$. Mais encore, la même année, à l'Exposition Universelle de Paris où Carrà travaillait comme décorateur, on avait reconstruit un village du Dahomey «avec ses têtes réduites plantées sur des piques, ses rites magiques mis en scène par des indigènes vêtus de leurs masques et costumes $»^{10}$.

Tout ceci nous suggère d'étudier plus profondément ce que le futurisme doit en terme de style et de forme à la tradition figurative non occidentale. Mais c'est là un travail qui dépasse les limites et l'objet de cet essai. Il faut par ailleurs reconnaitre que l'appropriation des caractéristiques de l'art tribal de la part des futuristes fut un phénomène assez tardif. Pourquoi donc, dès 1910, les futuristes se proclamèrent-ils «les primitifs d'une nouvelle sensibilité totalement renouvelée »?

Dans la préface au catalogue de l'exposition parisienne de 1912, le «fondement du primitivisme futuriste » est défini comme une conception relative tant à la perception des rapports entre les objets qu'aux relations entre sujet et objet et s'oppose diamétralement au «fondement du primitivisme impressionniste $»^{11}$. Deux ans après, Boccioni allait développer ce rapport entre futurisme et impressionnisme dans son écrit théorique le plus important. Il reconnaissait aux impressionnistes le double mérite d'avoir «ouvert la voie à une identité réelle et moderne entre intérieur et extérieur» et d'avoir «à la fois conquis et créé un nouveau corps, l'atmosphère ». Il précisait: «Pour la première fois, un objet vit et se complète avec le milieu en donnant et en recevant des influences $\gg^{12}$. Boccioni voyait donc chez les impressionnistes les précurseurs des deux principes fondamentaux de l'art futuriste: la poétique des états d'âme et la compénétration des plans. Toutefois, pour Boccioni, les expériences des impressionnistes étaient destinées à rester «des fragments esclaves de la vérité comme conception et donc objectifs et limités comme interprétation $\gg^{13}$; par ailleurs il estimait que « la négation violente de la fantaisie et du mystère » et la frénésie expérimentale qui poussait les impressionnistes à immortaliser de «fugitifs instants lumineux » avaient fait en sorte que « l'apparence prît la place de la réalité » ${ }^{14}$.

Pour revenir à la préface de 1912, on comprendra désormais l'affirmation selon laquelle «chaque objet influence son voisin, non par des réflexions de lumière (fondement du primitivisme impressionniste) mais par une réelle concurrence de lignes et de réelles batailles de plans, en suivant la loi d'émotion qui gouverne le tableau (fondement du primitivisme futuriste) $\gg^{15}$.

On retrouve déjà préfiguré ici, par rapport à la comparaison avec les impressionnistes, le principe de la compénétration des plans (nous aurons l'occasion de revenir sur celui-ci) et la poétique des états d'âme (figs.1, 2 et 3). Il nous suffit de souligner ici l'insistance avec laquelle les futuristes affirment le caractère réel, non apparent, non conventionnel et non arbitraire de la concurrence des lignes et des batailles des plans qu'ils entendent représenter. L'œuvre d'art n'est pas imitation illusoire de l'apparence des choses mais réification et, en tant que telle, révélation de la plus intime essence du réel à laquelle une « loi d'émotion », et

\footnotetext{
${ }^{9}$ Voir l'autobiographie de C. Carrà, «La mia vita », in id., Tutti gli Scritti, op.cit., p. 620 [1 1ère éd. 1945].

${ }_{10}$ Voir M. G. Messina, Le Muse d'Oltremare, op.cit., p. 172. Il faut pourtant préciser que, aussi bien en ce qui concerne l'influence de Modigliani, supposée par Messina, que dans le cas des pièces exposées au Musée du Trocadéro, les suggestions de l'art tribal parvinrent à Carrà à travers le filtre de la sensibilité et du goût occidentaux. Sur la médiation que la tradition artistique occidentale exerça sur la réception de l'art tribal en Europe, voir C. Ginzburg, «Oltre l'esotismo : Picasso e Warburg », in id., Rapporti di Forza, Milan, Feltrinelli, 2000, p. 127-147.

11 «Les exposants au public », op.cit.

${ }^{12}$ U. Boccioni, Dynamisme Plastique : Peinture et sculpture futuristes, (texte établi, annoté et préfacé par G. Lista, tr. C. Minot et G. Lista), Lausanne, L’Age d'Homme, 1975, p. 48. Voir U. Boccioni, « Pittura scultura futuriste (Dinamismo plastico)», in id., Scritti Editi e Inediti, op.cit., p. 114 [1 ère éd. 1914].

${ }^{13}$ Ibid., p. 47 [p. 114].

${ }_{14}$ Ibid., p. 48 [p. 116].

15 «Les exposants au public », op.cit., p. 7.
} 
non l'aride déchiffrement intellectualiste, permettrait d'accéder. La participation émotive devient la prémisse indispensable de l'interprétation de l'œuvre d'art :

Le désir d'intensifier l'émotion esthétique fondant en quelque sorte la toile peinte avec l'âme du spectateur, nous a fait déclarer que celui-ci "doit être placé désormais au centre du tableau”. Il n'assistera pas, mais il participera à l'action. Si nous peignons les phases d'une émeute, la foule hérissée de poings et les bruyants assauts de la cavalerie se traduisent sur la toile par des faisceaux de lignes correspondant à toutes les forces en conflit, en suivant la loi de violence générale du tableau. Ces lignes-forces doivent envelopper et entraîner le spectateur qui sera en quelque sorte obligé de lutter lui aussi avec les personnages du tableau ${ }^{16}$.

Telles des tentacules inexorables, les forces qui se dégagent de la toile capturent le spectateur et l'entraînent dans une expérience sensorielle révélatrice. Pour y accéder, le public devrait «oublier complètement sa culture intellectuelle, non pas pour s'emparer de l'œuvre d'art, mais pour se livrer à elle éperdument $»^{17}$. Le rejet de la tradition mimétique propre à l'art occidental ${ }^{18}$ allait de paire avec la revendication d'une palingénésie artistiquespirituelle du public: "pour concevoir et comprendre les beautés nouvelles d'un tableau moderne, l'âme doit redevenir pure et l'œeil doit se libérer du voile dont l'atavisme et la culture l'ont recouvert ${ }^{19}$. Et la récupération des expressions artistiques propres aux époques archaïques ou aux cultures non-occidentales aurait pu contribuer à une telle palingénésie de façon efficace: «notre devoir est de détruire quatre siècles de tradition italienne, d'introduire dans le vide qui peut en résulter tous les germes de puissance qui sont dans les exemples des primitifs et des barbares de chaque pays $»^{20}$.

Le retour au primitivisme ne revêtait pas, cependant, aux yeux de Boccioni, une valeur purement critique et négative : «l'exemple des primitifs » était pour lui si puissant parce qu'il pensait que cette même «loi d'émotion» à laquelle les futuristes affirmaient obéir gouvernait également l'art archaïque et tribal. Il affirmait :

Plus on remonte aux époques antérieures, moins on trouve l'obsession pitoyable de la duperie optique qui semble être l'une des armes les plus fortes et les plus utilisées pour nous combattre. La peinture et la sculpture des époques primordiales se préoccupent de suggestionner et de suggérer et elles le font grâce à n'importe quel moyen sans alluder aucunement au stupide exercice artistique toujours en dehors de la réalitée ${ }^{21}$.

La distinction entre art et réalité, abolie par les futuristes, n'existait pas dans les cultures des « époques primordiales ». Boccioni écrivait :

Au cours de ces heureuses époques, on ne connaissait ni le mot art, ni le concept d'artistique, ni encore les subdivisions artificielles telles que la peinture, la sculpture, la musique, la littérature, la poésie, la philosophie... $\mathrm{Au}$ contraire, tout est architecture car tout, dans l'art, doit être création d'organismes autonomes construits avec des valeurs plastiques abstraites, c'est-à-dire avec les équivalents de la réalitée ${ }^{22}$.

\footnotetext{
16 Ibid., p. 8.

${ }_{17}$ Ibid., p. 10.

18 «Tout est conventionnel en art. Ce qui était une vérité pour les peintres d’hier n’est plus qu'un mensonge aujourd'hui. Nous déclarons par exemple qu'un portrait ne doit pas ressembler à son modèle, et que le peintre porte en soi les paysages qu'il veut fixer sur la toile » («Manifeste des peintres futuristes », op.cit., p. 163-164). Voir « Pittura futurista. Manifesto tecnico », op.cit., p. 7.

19 Ibid., p. 9.

${ }^{20}$ U. Boccioni, « Fondamento plastico », op.cit., p. 39.

${ }^{21}$ Ibidem.

${ }^{22}$ Ibidem. Je ne suis pas en mesure d'établir d'où Boccioni tirait cette interprétation de l'art primitif ou envers quels auteurs elle était débitrice. On pourrait se demander si le peintre connaissait des textes d'anthropologie culturelle. On peut supposer qu'il ait eu l'occasion d'en discuter avec son beau-frère Guido Valeriano Callegari, éminent spécialiste d'antiquités amérindiennes, mais aucune évidence ne confirme cette hypothèse. À ce propos il faut d'ailleurs rappeler, comme Raymond Firth l'a remarqué, qu'«avant la Première Guerre Mondiale, et
} 
William Rubin a affirmé que la fascination que l'art tribal exerçait sur les artistes d'avant-garde $d u$ début du xxème siècle serait à relier principalement «à un passage fondamental de la plupart des avant-gardes artistiques de styles enracinés dans la perception visuelle vers des styles fondés sur la conceptualisation $»^{23}$. Ernest Gombrich à son tour a suggéré d'interpréter «la révolution primitiviste» comme "une réaction au faux art des virtuoses à succès $»^{24}$. Ces deux explications mettent en cause des facteurs qui jouèrent un rôle important dans la récupération de la culture figurative des peuples tribaux par les futuristes. Il nous suffit de penser ici à la vieille polémique qu'ils engagèrent contre la tradition mimétique de l'art occidental et au rejet de l'« harmonie traditionnelle qui nous fait tomber dans un gracieux fabriqué grâce à de honteux artifices sentimentaux », pour citer de nouveau l'éloquent Boccioni ${ }^{25}$.

Toutefois, ni l'interprétation de Rubin ni celle de Gombrich ne réussissent à rendre pleinement compte du primitivisme futuriste. Car, il ne se résout pas tant dans la mutation des formes synthétiques ou grotesques fonctionnelles à un processus de conceptualisation ou à la réaction contre le beau maniéré que dans la récupération d'un rapport entre art et réalité. Les futuristes considéraient ce dernier (à tort ou à raison) propre à l'art archaïque ou tribal qui, selon eux, ne représentait pas le visible mais permettait de pressentir l'invisible à travers la participation émotionnelle et l'abandon extatique : « Nous avons l'extase du moderne et le délire innovateur de notre époque. Nous, peintres futuristes, nous avons pour cette extase et ce délire une force psychique divinatoire donnant à nos sens la possibilité de percevoir ce qui jusqu’à présent n’avait jamais été perçu »26.

Fig.1.

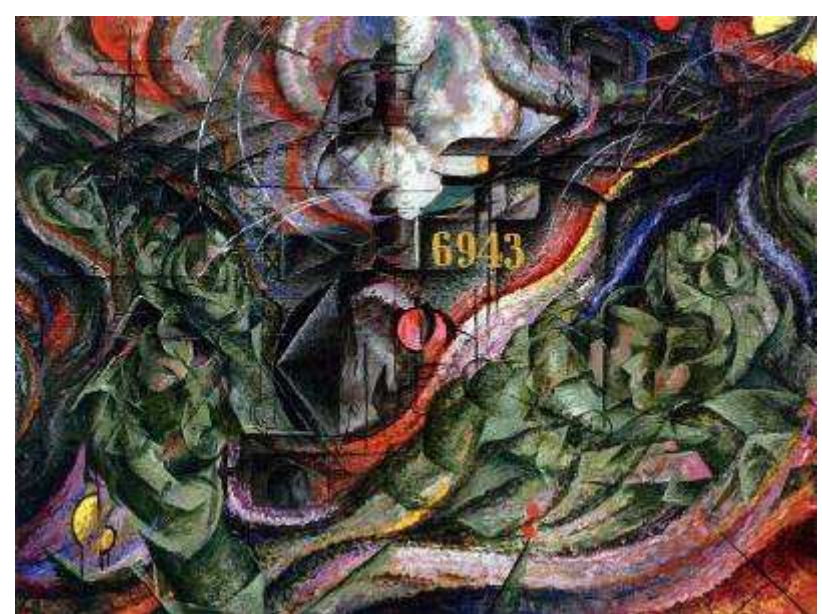

Umberto Boccioni, Les Etats d'Ame. Les Adieux, 1911

Huile sur toile, 70,5 x 96,2 cm, New York, The Museum of Modern Art

encore entre les deux guerres, l’intérêt de l'anthropologie pour l'art était très limité et le développement de la connaissance de ce qu'on appelle l'art primitif était du essentiellement à une combinaison des préoccupations de nature esthétique et commerciale, et non pas ethnographique ». Comme on le sait, la première œuvre systématique sur l'art primitif écrite par un anthropologue fut Primitive Art de Franz Boas en 1927. Voir R. Firth, "Art and anthropology», in J. Coote et A. Shelton (dir.), Anthropology, Art and Aesthetic, Oxford, Oxford University Press, 1992, p. 20.

23 "With a fundamental shift in the nature of most vanguard art from styles rooted in visual perception to others based on conceptualization", l'opinion de Rubin est rapportée par E. H. Gombrich, The Preference for the Primitive. Episodes in the history of Western taste and art, Londres, Phaidon, 2002, p. 201.

${ }_{24}^{2}$ «A reaction against the meretricious art of successful virtuosus » (ibid., p. 203).

${ }^{25}$ U. Boccioni, « Fondamento plastico », op.cit., p. 40.

26 « Nous pensons, poursuivait Boccioni, que, si tout tend à l'unité, ce que l'homme a toujours rêvé de concevoir en unité n'est qu'une misérable, aveugle et puérile subdivision des choses. À notre avis, la science a ramené les hommes à une sorte de barbarie, à une merveilleuse barbarie supérieure qui nous rend assoiffés de réalité et écœurés par des apparences artistiques de tous ordres » (U. Boccioni, Dynamisme plastique, op.cit., p. 32). Voir U. Boccioni, « Pittura scultura futuriste », op.cit., p. 89. 
Fig.2.

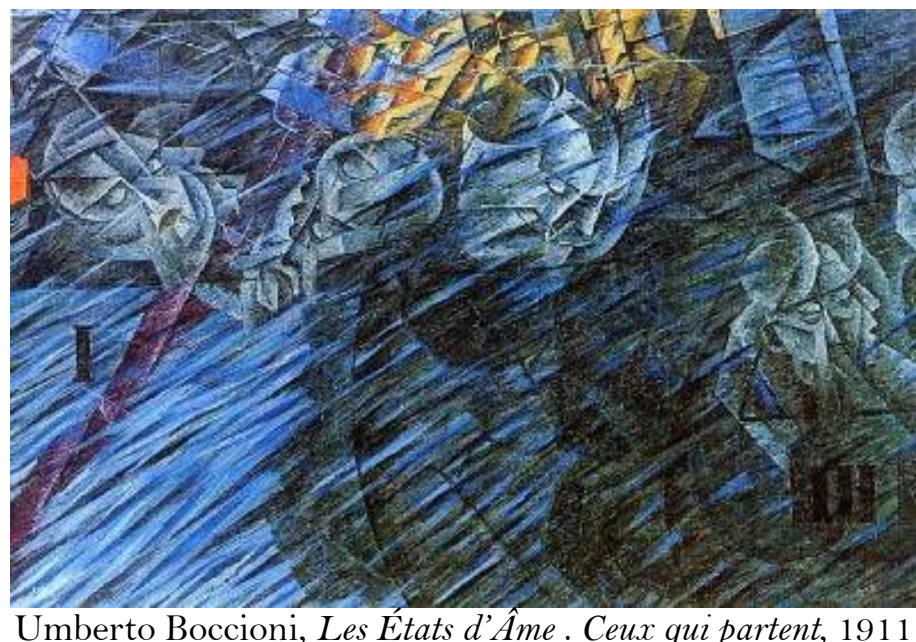

Huile sur toile, 70,8 x 95,9 cm, New York, The Museum of Modern Art

Fig.3.

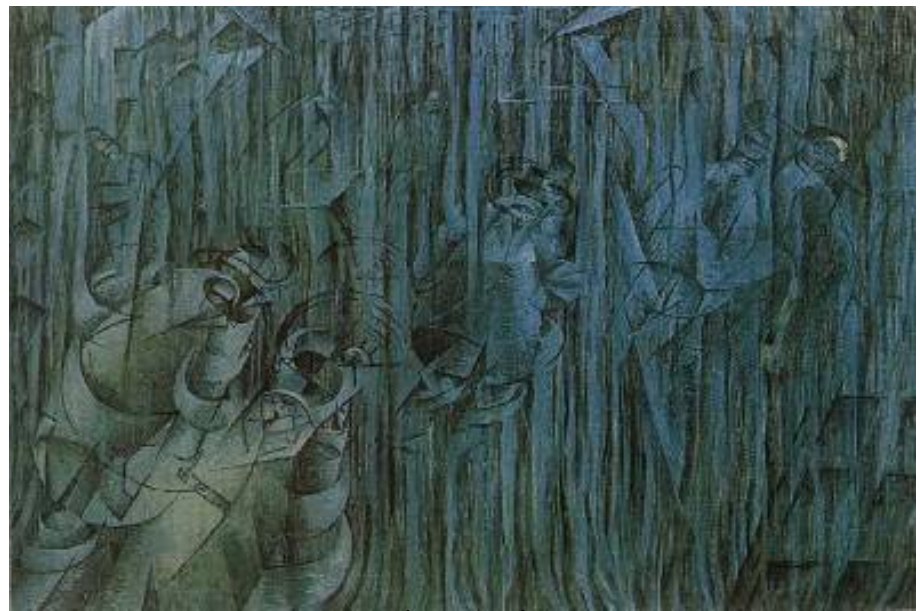

Umberto Boccioni, Les Etats d'Áme. Ceux qui restent, 1911

Huile sur toile, 70,8 x 95,9 cm, New York, The Museum of Modern Art

2.

Il faut donner l'invisible qui agit et qui vit au-delà des épaisseurs, ce que nous avons à droite, à gauche et derrière nous et non pas le petit carré de vie artificiellement serré comme entre les décors d'un théâtre ${ }^{27}$.

C'est ce qu'affirmaient les peintres futuristes dans le catalogue de l'exposition parisienne de 1912. En refusant la «construction des tableaux [...] stupidement traditionnelle » au moyen de laquelle « les peintres nous ont toujours montré les objets et les personnes placés devant nous ${ }^{28}$, les futuristes se proposaient de représenter la " simultanéité des états d'âme ", c'est-à-dire la totalité d'une expérience sensorielle. Figurer les invisibles signifiait donc pour les futuristes tout d'abord rompre les liens matériels qui contraignaient l'artiste à sélectionner arbitrairement un fragment de réel et à concevoir la

27 «Les exposants au public », op.cit., p. 6.

28 «Manifeste des peintres futuristes », op.cit., p. 164. Voir « Pittura futurista », op.cit., p. 9. 
peinture comme une pure réplique mimétique de ce fragment. Réintégrer dans l'œuvre d'art ce qui en était d'habitude supprimé impliquait non seulement de représenter ce qui se trouvait devant l'artiste mais aussi derrière lui et de traduire en image des sensations auditives et tactiles au même titre que les sensations visuelles. Cela voulait dire aussi tenter une "synthèse de ce dont on se souvient et de ce que l'on voit », c'est-à-dire abolir les barrières temporelles et condenser sur la toile passé, présent et futur. Les futuristes écrivaient: «Nous avons déclaré dans notre manifeste qu'il faut donner la sensation dynamique, c'est-à-dire le rythme particulier de chaque objet, son penchant, son mouvement, ou, pour mieux dire, sa force intérieure ». Ainsi les lignes-force devaient-elles traduire sur la toile les trajectoires et le ton de ce rythme. Les futuristes précisaient: « chaque objet révèle par ses lignes comment il se décomposerait s'il suivait les tendances de ses forces $»^{29}$.

Le concept des lignes-force était au cour de la théorie du transcendantalisme physique dont Boccioni avait donné une première définition au cours de la Conférence sur la peinture futuriste qu'il avait tenue au Cercle artistique de Rome en mai 1911. Dans le premier Manifeste Technique de la Sculpture Futuriste (11 avril 1912), Boccioni expliquait: «cette vision que j'ai appelée transcendantalisme physique (au cours de la Conférence sur la peinture futuriste que j'ai tenue au Cercle artistique de Rome en mai 1911) pourra rendre plastiques les affinités mystérieuses que créent les influences formelles réciproques des plans des objets $»^{30}$.

Il ne s'agit pas, comme tout le monde le croit, de faire une peinture abstraite..., précisait-il quelques années après, il s'agit, au-delà de ça, de réaliser et de rendre plastique et concret, à travers un raffinement de la sensibilité, ce qui jusque là était considéré comme immatériel, infaçonnable, invisible ${ }^{31}$.

Représenter l'invisible ne signifiait donc pas seulement représenter ce qui échappait à une traditionnelle position frontale du peintre par rapport au tableau (c'est-à-dire ce qui se trouvait derrière son dos) ni représenter des contenus non immédiatement manifestes selon un processus symbolique ou métaphorique : les lignes-force constituaient la concrétisation sur la toile des forces occultes qui gouvernaient la réalité. On comprendra désormais pleinement les implications du concept de primitivisme futuriste qui a été analysé dans le paragraphe précédent.

Dans Über das Geistige in der Kunst (la première édition date de 1912), Kandinsky exposait une conception tout à fait analogue des rapports entre art et réalité. Comme l'a observé Ringbom, ce traité reflétait l'interprétation qu'avait donnée Rudolf Steiner des notions de Goethe sur l'art. Pour Steiner, Goethe aurait éliminé « la dichotomie platonique entre Nature et Art », ce qui posait un terme à la conception de la création artistique comme forme supérieure de Naturwirken: «L'artiste réalise l'idée de nature, il montre à quoi la Nature ressemblerait si ses forces intérieures étaient accessibles non seulement à la pensée spéculative mais aussi à la perception directe $»^{32}$.

Comme on le sait, Rudolf Steiner, chef de la section allemande de la Société Théosophique et fondateur de la Société Anthroposophique, fut l'un des protagonistes du retour de l'occulte sur la scène culturelle européenne au cours des dernières années du XIXème siècle.

\footnotetext{
29 «Les exposants au public », op.cit., p. 7.

30 U. Boccioni, «Manifeste technique de la sculpture futuriste (1912)», in G. Lista, Futurisme, op.cit., p. 173. Voir U. Boccioni, «Manifesto tecnico della scultura futurista», in U. Boccioni, Scritti Editi e Inediti, op.cit., p. 24.

${ }^{31}$ U. Boccioni, Dynamisme Plastique, op.cit., p. 78. Voir U. Boccioni, « Pittura scultura futuriste », op.cit., p. 158.

32 «The artist realizes the ideas of nature, he demonstrates what Nature would look like if its inherent forces were accessible, not only for speculative thought, but to direct perception » (S. Ringbom, «Art in the Epoch of the Great Spiritual. Occult elements in the early theory of abstract painting »; Journal of the Warburg and the Courtauld Institutes 29, 1966, p. 390).
} 
3.

Il existe de nombreux témoignages de l'intérêt que Boccioni et d'autres représentants du futurisme nourrirent pour l'occultisme - généralement entendu comme « une attitude envers le monde qui emphatise les aspects cachés ou secrets de la réalité ${ }^{33}$ ). On sait, par exemple, que l'intérêt du père de Marinetti pour les religions orientales, et en particulier pour le phénomène de la télékinésie, influença profondément l'éducation de son fils ${ }^{34}$. Les expériences d'Anton Giulio Bragaglia, au début des années 1910, sur les potentialités de la technique photographique dans la représentation des phénomènes médiumniques sont bien connues. Il en rendit compte dans deux articles parus entre novembre et décembre 1913 dont les titres évocateurs sont Les fantômes des vivants et des morts et La photographie de $l^{\prime}$ invisible ${ }^{35}$. Si Gino Severini prit part à des séances de spiritisme avec sa femme Jeanne, Luigi Russolo fut sans aucun doute, parmi les peintres futuristes, le plus fasciné par les sciences de l'occulte. Le tableau Autoportrait avec double éthéré, dont la première version remonte à $1910^{36}$, mais aussi le volume Au-delà de la matière (publié par les frères Bocca en 1938) dans lequel l'artiste résuma ses pensées mystiques et philosophiques, en témoignent ${ }^{37}$. Quant à Boccioni, il reconnut son intérêt de longue date pour les phénomènes médiumniques dans un article publié dans Lacerba en 1913. Il y parle de : "la perception de phénomènes similaires jusqu'ici inconnus de notre sensibilité obtuse, comme les perceptions d'émanations lumineuses de notre corps dont j'ai parlé lors de ma première conférence à Rome et que la plaque photographique est en mesure de reproduire $»^{38}$. Dans Dynamisme Plastique il soutenait: «le mystère biologique de la matérialisation médiumnique est pour nous une certitude ${ }^{39}$. Par ailleurs, dans le Manifeste Technique de la Peinture Futuriste de 1910, les futuristes se demandaient déjà : «qui donc peut croire encore à l'opacité des corps, du moment que la sensibilité aiguisée et multipliée a déjà deviné les obscures manifestations de la médiumnité ? »40.

Certains chercheurs qui ont étudié les rapports entre futurisme et occultisme se sont limités à comparer les analogies significatives entre les convictions artistiques des futuristes relatives à la dématérialisation des corps, à la compénétration des plans ou aux forces intérieures qui déterminent le mouvement des objets et la façon dont les sciences occultes ont expliqué les supposés phénomènes médiumniques. Mais d’autres sont allés jusqu'à faire l'hypothèse selon laquelle les solutions particulières adoptées par les futuristes sur les plans

${ }^{33}$ La définition est proposée par R. Galbreath dans A Glossary of Spiritual and Related Terms, in M. Tuchman (éd.), The Spiritual in Art. Abstract Painting 1890-1985, Los Angeles-County, Museum of Art, New YorkAbbeville Press, 1986, p. 380. À propos des intérêts des futuristes pour l'occultisme, voir: G. Celant, «Futurism and the occult », Artforum 19, janvier 1981, p. 26-42 ; G. Lista, «Futurismus und Okkultismus », in V. Loers (éd :), Okkultismus und Avantgarde von Munch bis Mondrian 1900-1915, Ostfildern ; Ed. Tertium, 1995, p. 431-444; S. Cigliana, Futurismo Esoterico. Contributi per una storia dell'irrazionalismo italiano tra otto $e$ novecento, Naples, Liguori, 2002 [1996].

${ }^{34}$ F. T. Marinetti, La Grande Milano Tradizionale e Futurista, (a cura di L. De Maria), Milan, Mondadori, 1969 ; G. Celant, «Futurism and the occult», op.cit., p. 7 ; G. Lista, «Futurismus und Okkultismus », op.cit., p. 435438.

35 A. G. Bragaglia, «I fantasmi dei vivi e dei morti », La Cultura Moderna 1 novembre 1913, p. 756-64 ; «La fotografia dell'invisible », Humanitas 21 décembre 1913, article repris avec quelques ajouts dans La Fotografia Artistica décembre 1913-janvier 1914, et dans A. G. Bragaglia, Fotodinamismo Futurista, 2ème éd. augmentée, Turin, Einaudi, 1980, p. 247-255. Une analyse des expériences de Bragaglia et des articles cités est conduite par M. Braun, «Fantasmes des vivants et des morts. Anton Giulio Bragaglia et la figuration de l'invisible », Études Photographiques 1, novembre 1996.

${ }^{36}$ Le tableau, dont la collocation n'est pas connue, est reproduit dans Okkultismus und Avantgarde, op.cit., p. 439.

${ }_{37}$ G. Severini, Al di là della Materia, Turin, Bocca, 1938.

${ }^{38}$ U. Boccioni, « Fondamento plastico », op.cit., p. 40.

${ }^{39}$ U. Boccioni, Dynamisme Plastique, op.cit., p. 105. Voir U. Boccioni, « Pittura scultura futuriste », op.cit., p. 202.

40 « Manifeste des peintres futuristes », op.cit., p. 164. Voir « Pittura futurista », op.cit., p. 7-8. 
stylistique et formel dépendraient directement de ces phénomènes et théories. Germano Celant a suggéré une analogie entre les processus de «condensation des énergies psychiques » qui, selon les théories spirites, donneraient lieu à l'apparition des fantômes et ectoplasmes, et les «traces » ou « images résiduelles » du mouvement que la recherche futuriste sur les mouvements de la matière concrétise. Ainsi, la Lampe à Arc de Balla ${ }^{41}$ (fig.4) pourrait-elle être définie, pour Celant, comme "presqu'un ectoplasme de lumière concrétisé $\gg^{42}$.

Fig.4.

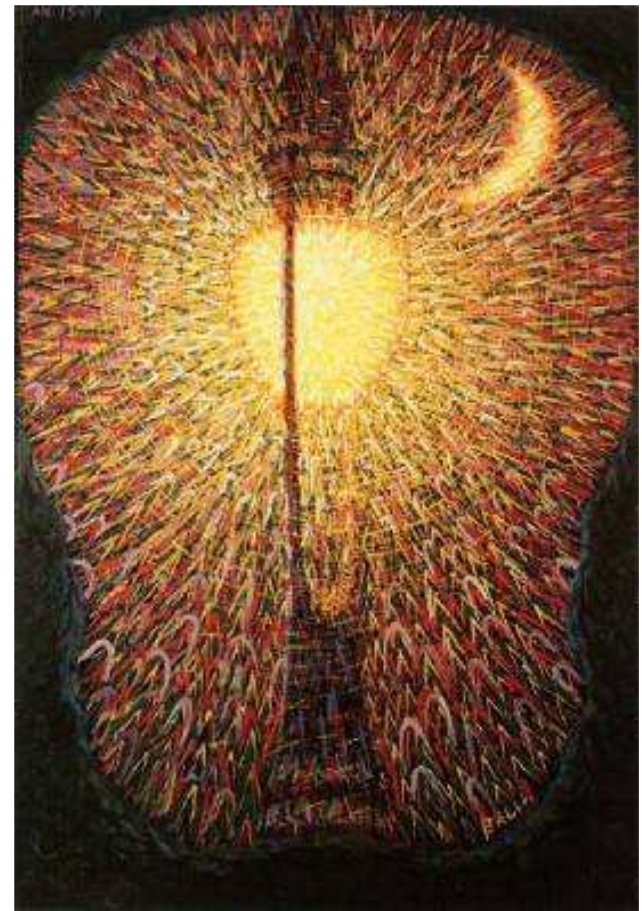

Giacomo Balla, Lampe à Arc, 1910-1911

Huile sur toile, 174,7 x 114,7 cm, New York, The Museum of Modern Art

Aucun écrit n'étaie l'interprétation de Celant. Du reste, si, comme il est bien connu, l'intérêt pour l'occultisme ne fut pas une particularité du futurisme mais bien un point commun entre les principaux représentants des avant-gardes européennes, du Symbolisme jusqu'au Cubisme, de l'Expressionisme jusqu'à l'Art abstrait en passant par le Surréalisme ${ }^{43}$, évoquer un tel intérêt pour rendre raison des particularités du style futuriste ne suffit pas. L'analyse qui va suivre permettra de dégager une articulation moins immédiate et plus circonstanciée du lien qui existe entre occultisme et expérimentation formelle futuriste, mais aussi de la relation qui unit l'occultisme et la poétique futuriste qui trouve l'une de ses principales inspirations dans la fascination pour la vie des métropoles modernes.

Enfin, l'affinité qui existe entre les théories métapsychiques et les concepts porteurs de la réflexion et de l'expérimentation artistique futuriste (comme les états d'âme, la compénétration des plans et le transcendantalisme physique) est un fait mais ne constitue pas à elle seule une raison suffisante pour expliquer pourquoi l'occultisme fit tant d'adeptes parmi les futuristes. Pour répondre à cette question, il faudra reconstruire les expériences

\footnotetext{
${ }^{41}$ Huile sur toile, 1909, New York, The Museum of Modern Art.

42 « Almost an objectified ectoplasm of light» (G. Celant, « Futurism and the occult », op.cit., p. 9-10).

${ }^{43}$ Voir L. Dalrymple Henderson (éd.), Mysticism and Occultism in Modern Art, numéro monographique de Art Journal 46(1), printemps 1987, The Spiritual in Art. Abstract Painting 1890-1985, op.cit., et Okkultismus und Avantgarde, op.cit.
} 
sociales partagées ${ }^{44}$ desquelles naquîrent la recherche formelle futuriste mais encore les conceptions sociales et politiques qui en découlèrent. Ceci nous permettra, en dernière analyse, de comprendre plus précisément quelle contribution les sciences de l'occulte ont apporté à la définition du langage artistique futuriste mais aussi l'articulation qui existe entre inspiration moderniste et survivances magiques.

4.

Le 5 février 1912 fut inaugurée à la galerie Bernheim-Jeune l'exposition Les Peintres Futuristes Italiens. Comme on le constate si on lit les journaux et revues d'alors mais aussi le témoignage de Carlo Carrà ${ }^{45}$, quatre toiles en particulier se distinguèrent parmi les trentesix exposées et retinrent l'attention des critiques à cause de leurs dimensions considérables : La Ville qui monte de Boccioni (199,3 x $301 \mathrm{~cm})^{46}$ (fig.5), Les Funérailles de Galli l'Anarchiste de Carrà (198,7 x 259,1c, $)^{47}$ (fig.6), La Révolte de Russolo (150 x 230cm) ${ }^{48}$ (fig.7) et La Danse du Pan Pan au Monico de Severini (280 x $400 \mathrm{~cm})^{49}$ (fig.8).

Fig.5.

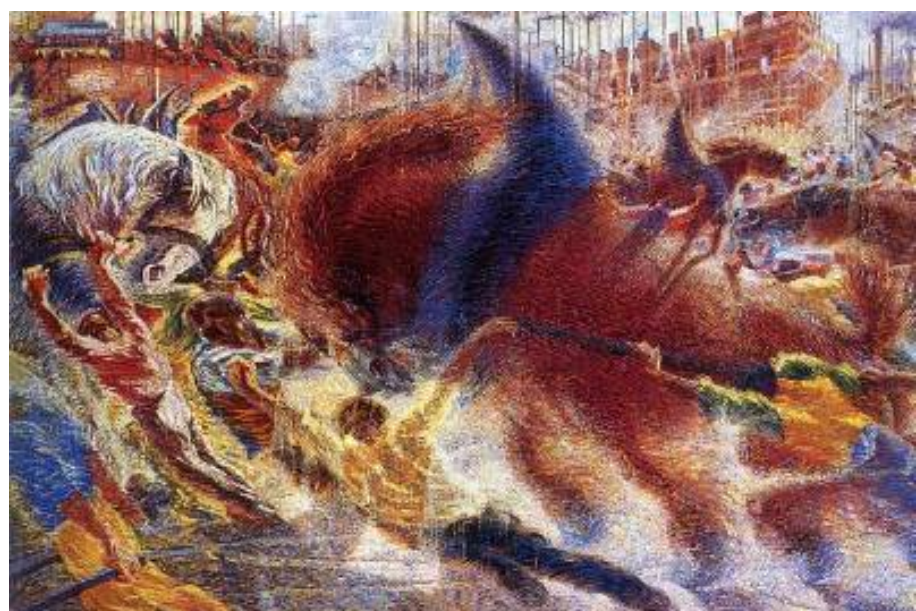

Umberto Boccioni, La Ville qui monte, c. 1910

Huile sur toile, 199,3 x $301 \mathrm{~cm}$, New York, The Museum of Modern Art

\footnotetext{
${ }^{44}$ Je me rapporte ici à la notion d'expérience sociale formulée par Michael Baxandall dans son ouvrage Painting and Experience in Fifteenth-Century Italy, Oxford, Oxford University Press, 1972 (tr. fr. L'CEil du Quattrocento. L'Usage de la Peinture dans l'Italie de la Renaissance. [tr. de l'anglais par Y. Delsaut], Paris, Gallimard, 1994). Baxandall y explore la relation entre le style pictural du Xvème siècle et les «expériences sociales partagées 》 en vertu desquelles le public était capable de le déchiffrer. $\mathrm{Si}$ «l'analyse des aspects pratiques et des conventions sociales peuvent aiguiser notre perception des tableaux », écrit-il, ainsi « les formes et les styles de la peinture peuvent aiguiser notre perception de la société »; voir ibid., p. 140.

45 Carrà rappelle: ces tableaux «soulevèrent de violentes discussions à notre première exposition de Paris (février 1912) », C. Carrà, «La pittura dei suoni, rumori e odori. Manifesto futurista (11 agosto 1913)», Lacerba (Florence) 1(17), 1 septembre 1913, et in Id., Tutti gli Scritti, op.cit., p. 21. Voir aussi N. Novelli, «Contributo a una bibliografia della fortuna del futurismo in Francia (1909-1920)», in La Fortuna del Futurismo in Francia, Rome, Bulzoni, 1979, p. 205-269.

${ }^{46}$ Huile sur toile, New York, The Museum of Modern Art (1910-1911).

${ }^{47}$ Huile sur toile, NewYork, The Museum of Modern Art (1910-1912).

${ }^{48}$ Huile sur toile, La Haye, Collection Gemeentemuseum Den Haag (1911).

${ }^{49}$ Huile sur toile, Paris, Centre Pompidou, Musée National d'Art Moderne (le tableau, peint avant 1912, fut détruit par les nazis et peint de nouveau par Severini en 1959-1960).
} 
Fig.6.

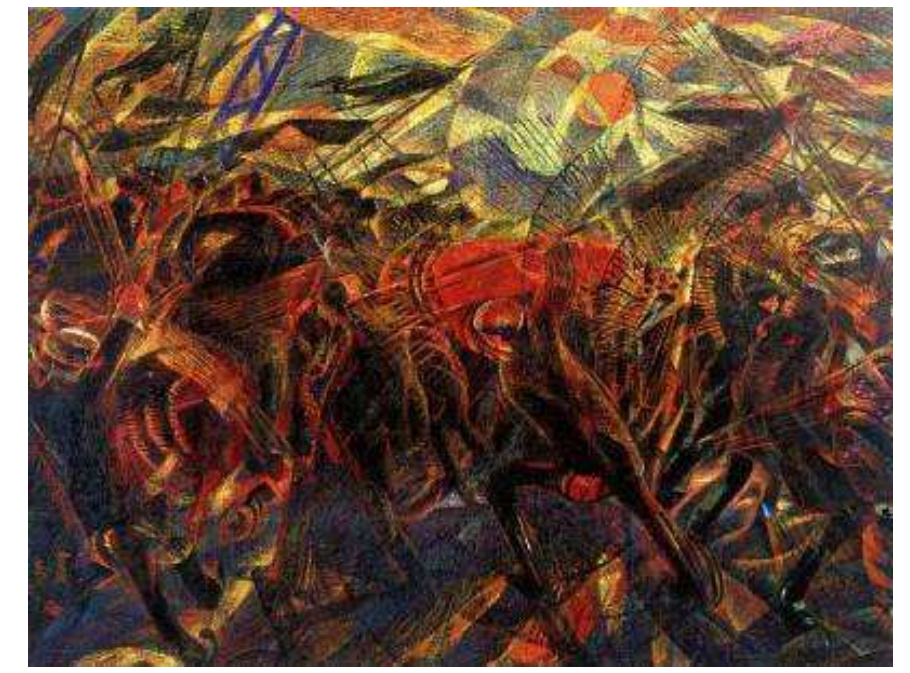

Carlo Carrà, Les Funérailles de Galli l'Anarchiste, 1910-1912

Huile sur toile, 198,7 x 259,1 cm, New York, The Museum of Modern Art

Fig.7.

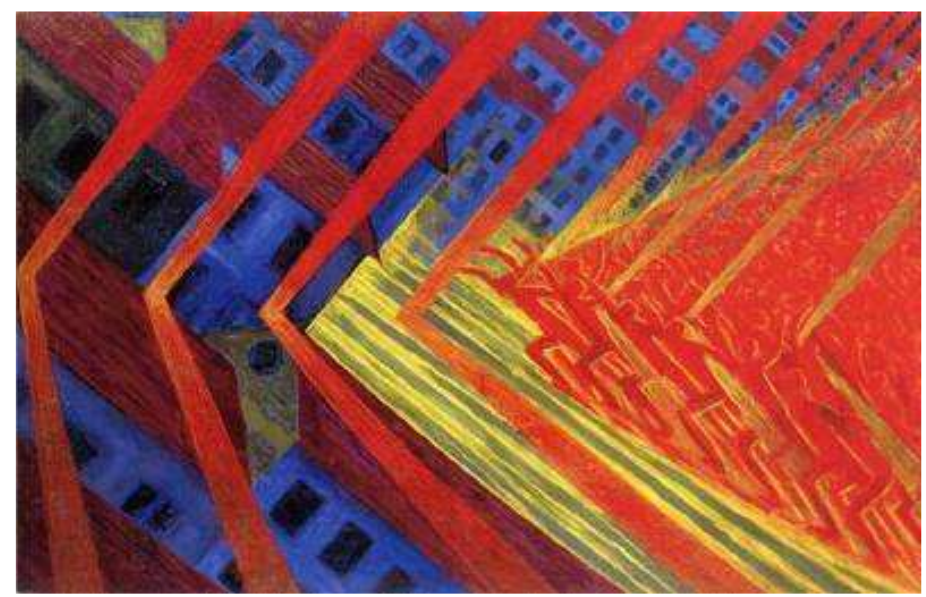

Luigi Russolo, La Révolte, 1911.

Huile sur toile, 150,8 x 230,7 cm, La Haye, Collection Gemeentemuseum Den Haag

Fig.8.

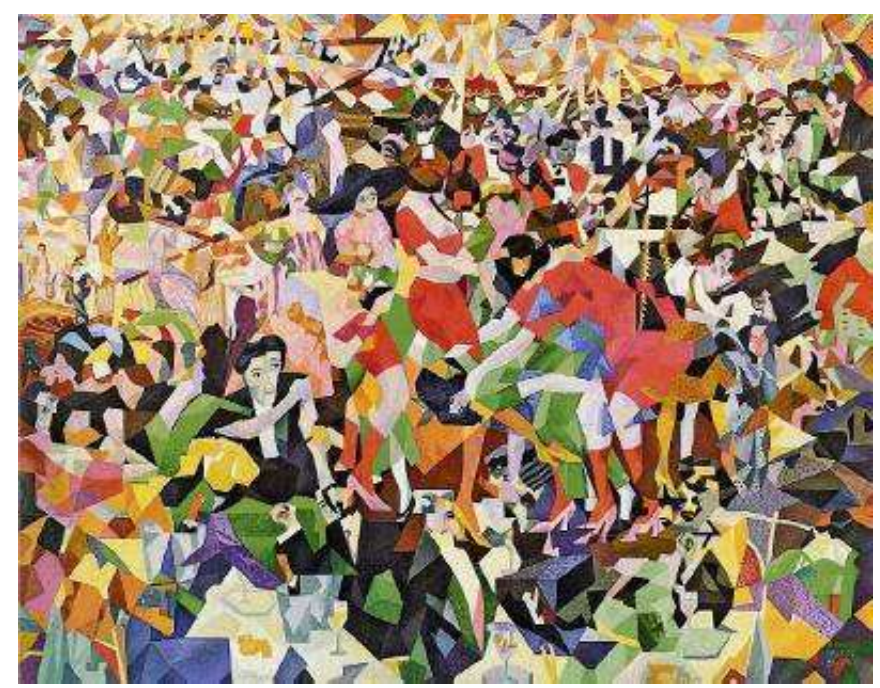

Gino Severini, La Danse du Pan-Pan au Monico, c. 1909-1911 (1959-1960)

Huile sur toile, $280 \times 400 \mathrm{~cm}$,

Paris, Centre Georges Pompidou, Musée National d'Art Moderne 
La critique a sans doute perçu le caractère emblématique que les peintres attribuaient à leurs œuvres. En effet, celles-ci étaient placées de façon significative (c'est ce que le catalogue nous révèle) à l'entrée des sections personnelles de chaque peintre qui articulaient l'exposition. Les historiens s'accordent pour reconnaître dans les quatre toiles la première tentative de condensation et de traduction en actes des principes de la peinture futuriste mise en ouvre par les auteurs du Manifeste Technique. Une analyse détaillée de la genèse et de la signification du tableau Les Funérailles de Galli l'Anarchiste nous permettra de mettre en lumière certains aspects thématiques et stylistiques de ces quatre toiles qui n'ont pas été jusqu'ici appréciés de façon adéquate.

Comme on le sait, le tableau représente les débordements qui eurent lieu au cours des funérailles d'Angelo Galli, ouvrier anarchiste tué au cours d'une grève générale en mai 1906. Carrà, qui assista à l'épisode, s'en souvenait ainsi dans ses mémoires :

La grève générale devait connaître par ailleurs un tragique dénouement avec la mort de l'anarchiste Galli rue Carlo Farini. Une fois porté le cadavre de Galli au cimetière de Musocco, les funérailles devaient avoir lieu, sur ordonnance de la police, sur la place en face du cimetière et, afin que cette ordonnance soit respectée, des cordons de police à cheval bloquaient les rues qui conduisaient à la ville. Mais les anarchistes décidèrent de s'y opposer et leur cortège, à la fin de l'avenue Sempione, attaqua soudainement les soldats qui leur répondirent avec une violence inouie. M'étant trouvé par hasard au cœur de la mêlée, je voyais devant moi le cercueil couvert d'œillets rouges ondoyer de façon menaçante sur les épaules des porteurs ; je voyais les chevaux s'emballer, les bâtons et les lances se rencontrer, de telle sorte qu'il me sembla que la dépouille allait d'un moment à l'autre tomber et que les chevaux allaient la piétiner. Très affecté, je me mis à dessiner le spectacle auquel j’avais assisté une fois rentré chez moi. Plus tard, je m'inspirai de ce dessin et d'autres qui le succédèrent pour réaliser mon tableau Les Funérailles de l'Anarchiste présenté ensuite à Paris, Londres et Berlin au printemps 1912 dans les expositions futuristes ${ }^{50}$.

Les évènements du 13 mai 1906 sont à la base de la réflexion artistique que Carrà allait approfondir au cours des années suivantes. "Très affecté » par la scène à laquelle il avait assisté malgré lui, Carrà en fit immédiatement un dessin (qui n'existe plus aujourd'hui) que l'on peut considérer comme la première déclinaison figurative d'un thème qui allait occuper durant les années à suivre ses méditation artistiques. Quand, un matin de février 1910, Carrà, Boccioni et Russolo, suite à une conversation électrisante avec Marinetti, se préparèrent à rédiger le Manifeste Technique de la Peinture Futuriste, les tragiques événements de 1906 revinrent à l'esprit de Carrà et l'amenèrent à formuler l'un des principes fondamentaux du nouvel art : «le souvenir de la scène dramatique, affirma-t-il, me poussa à écrire dans le manifeste de la peinture futuriste la phrase : 'nous placerons le spectateur au centre du tableau' $»^{51}$.

Ce témoignage nous amène à supposer que Carrà avait continué à penser à ces évènements au fil des années et plus précisément que cette expérience avait joué un rôle central dans la direction que prit son expérimentation artistique. En effet, c'est alors qu'il travaillait à ce sujet qu'il parvint à la définition d'un nouveau langage pictural proprement futuriste. Les chercheurs s'accordent pour situer le tournant décisif de l'élaboration du style de Carrà entre la première version de l'œuvre, présentée à l'Exposition d'Art Libre de Milan en avril 1911 et sa version définitive, résultat du remaniement de la première version que Carrà entreprit suite à son voyage à Paris en octobre 1911 et à sa rencontre avec le Cubisme. Du reste, si l'on compare de façon attentive le tableau et l'esquisse réalisée en $1910^{52}$ (fig.9), on constate un écart considérable entre les deux et cela nous permet de mettre en lumière les

\footnotetext{
${ }^{50}$ C. Carrà, « La mia vita », op.cit., p. 634.

51 Ibidem.

${ }_{52}$ Pastel sur carton, collection particulière.
} 
innovations fondamentales que Carrà introduisit dans ce tableau. L'utilisation des traits et des touches de couleur, révélatrice d'une technique encore ancrée dans le divisionnisme, est le seul élément qu'ont en commun le tableau et son dessin préparatoire, si on les observe bien. Dans la peinture, même si elle n'a été réalisée qu'à peu de mois du dessin, la structure de la composition originelle, équilibrée et étendue, est soumise à un mouvement de compression et de rotation centripète. Les figures, presque englouties dans un tourbillon, apparaissent déformées, allongées et mélangées et il est difficile d'en distinguer les contours et la position dans l'espace.

Fig.9.

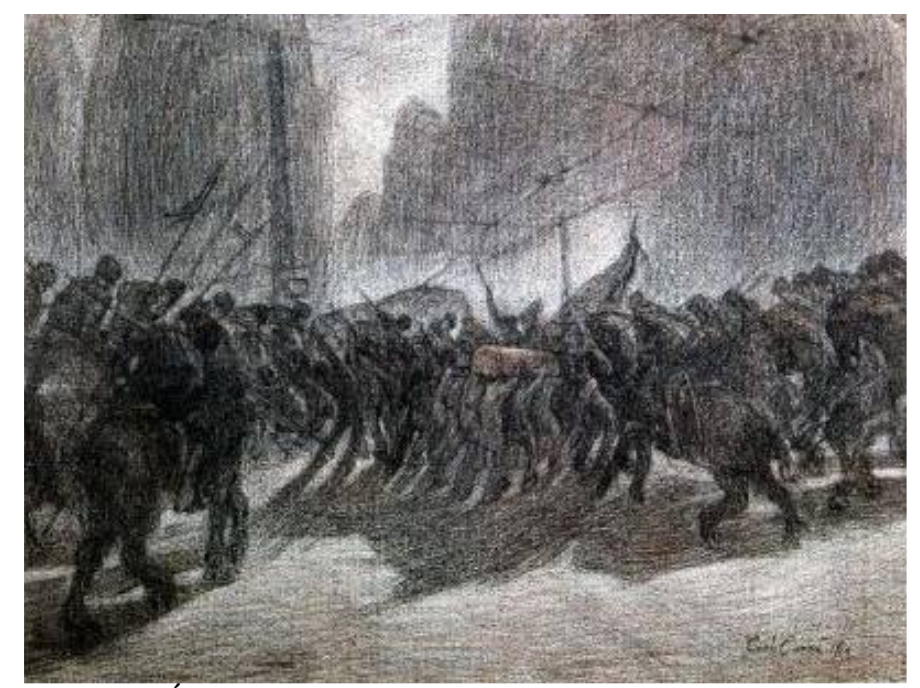

Carlo Carrà, Étude pour Les Funérailles de Galli l'Anarchiste, 1910

Pastel sur carton, 57 x $78 \mathrm{~cm}$, Collection particulière

À la condensation de la scène représentée correspond un considérable rapprochement du point de vue indiqué par la disparition du premier plan. L'adoption du point de vue rapproché et le renoncement à toute norme de composition traditionnelle contribuent à annuler la profondeur de la perspective. Malgré cela, l'observateur se sent irrésistiblement impliqué dans la scène représentée, lui aussi attiré dans le tourbillon qui entraîne hommes et animaux. On comprend bien pourquoi le tableau a pu être considéré dans l'introduction au catalogue de l'exposition parisienne comme l'une des mises en ouvre les plus réussies de ce principe de la peinture futuriste qui énonçait : « la construction des tableaux est stupidement traditionnelle. Les peintres nous ont toujours montré les objets et les personnes placés devant nous. Nous, nous placerons le spectateur au centre du tableau $»^{53}$. Comme on l'a vu, ce principe se corrélait fortement à son tour avec la définition du " primitivisme futuriste ».

Quant au sujet du tableau, il a sans doute transcendé l'épisode dont Carrà s'était inspiré. Dans la préface au catalogue de l'exposition parisienne, la foule hérissée de poings qui soutient le bruyant assaut de la cavalerie est indiquée comme la protagoniste du tableau. Il émerge en outre de façon évidente du compte-rendu des évènements que Carrà fit trente ans plus tard dans son autobiographie, que ce qui l'impressionna le plus ne fut pas tant la dynamique des faits en eux-mêmes, pour autant dramatique qu'elle fut, mais bien l'expérience de se trouver « au centre de la mêlée ».

De plus, le fait que la foule apparaisse ou soit évoquée non seulement dans de nombreux tableaux de Carrà peints aux cours des années qui précédèrent la guerre ${ }^{54}$

\footnotetext{
${ }_{53}$ « Manifeste des peintres futuristes » (1910), op.cit., p. 164. Voir « Pittura futurista », op.cit., p. 9.

${ }^{54}$ On pense à Notturno a Piazza Beccaria (1910, Milan, Civiche Raccolte d'Arte, Museo del Novecento); Piazza del Duomo (1910, Milan, Collection particulière) (fig.10); Uscita da Teatro (1910-11, Londres, Estorick Collection) (fig.11). Ces deux derniers furent exposés à Paris avec Les Funérailles; Stazione a Milano (1910-
} 
(figs.10 à 13) mais aussi chez les autres peintres futuristes à la même époque ${ }^{55}$ (figs.14 à 17) confirme l'hypothèse selon laquelle la foule serait le thème qu'il entendait explorer quand il a peint Les Funérailles de Galli l'Anarchiste. Soulignons aussi que les trois autres tableaux de grandes dimensions que nous avons cités plus haut (La Ville qui monte de Boccioni, La Révolte de Russolo et La Danse du Pan-Pan au Monico de Severini) ont eux-mêmes la foule pour sujet.

Fig.10.

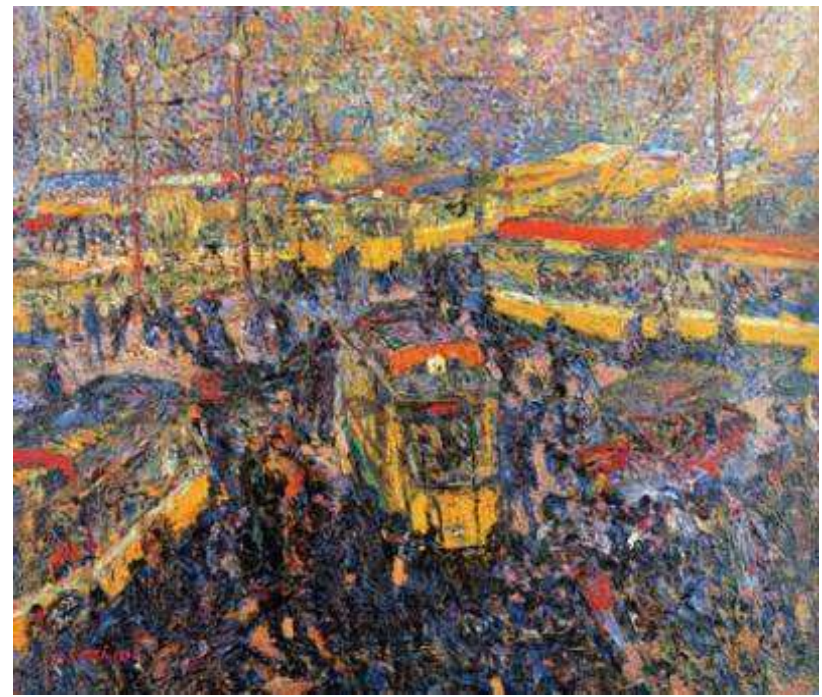

Carlo Carrà, Place du Duomo, 1910

Huile sur toile, 45 x $60 \mathrm{~cm}$, Milan, Collection particulière

Fig.11.

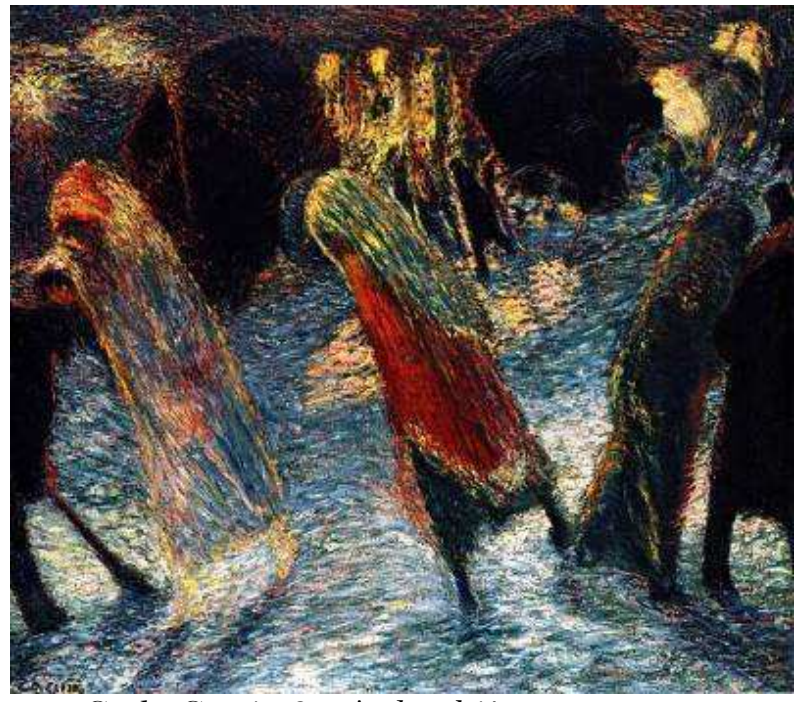

Carlo Carrà, Sortie de Théâtre, 1910-1911

Huile sur toile, 69 x $89 \mathrm{~cm}$, Londres, Eric and Salome Estorick Foundation

1911, Collection particulière) (fig.12); Ciò che mi ha detto il tram (1911, Rovereto, Museo d'arte moderna e contemporanea di Trento e Rovereto) (fig.13); Luci Notturne (1911, Collection particulière); La Galleria di Milano (1912, Venise, Collection Gianni Mattioli - dépôt temporaire collection Peggy Guggenheim).

${ }_{55}^{55}$ Pour Boccioni, on pense à Gli Stati d'Animo (1911, New York, The Museum of Modern Art), La Strada entra nella Casa (1911, Hannover, Sprengel Museum Hannover), La Città che sale (1910-1911, New York, The Museum of Modern Art), La Retata (1910, Collection particulière) (fig.14), exposés à Paris en 1912, ou encore à la célèbre Rissa in Galleria (1910, Milan, Pinacoteca di Brera) (fig.15), à Baruffa (1910, New York, The Museum of Modern Art) (fig.16) ou à une gravure moins connue intitulée Folla che Circonda un Monumento Equestre (1908, Birmingham, Collection Winston) (fig.17). 
Fig.12.

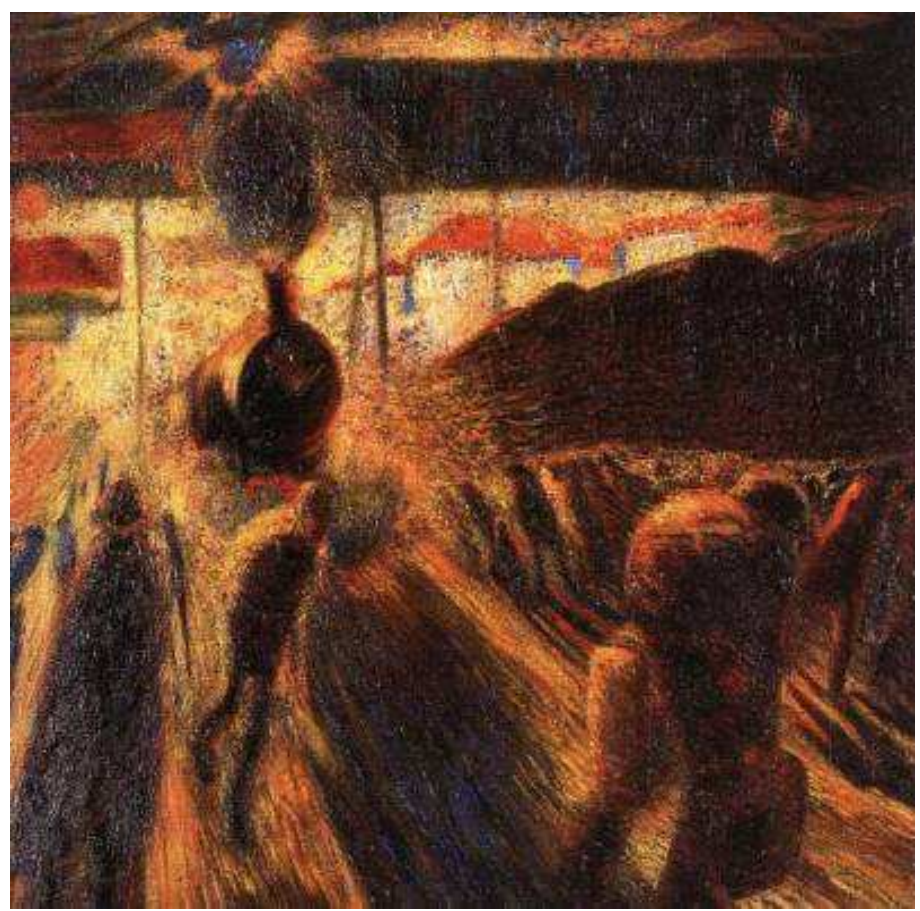

Carlo Carrà, Gare à Milan, 1910-1911

Huile sur toile, 80 x $90 \mathrm{~cm}$, Collection particulière

Fig.13.

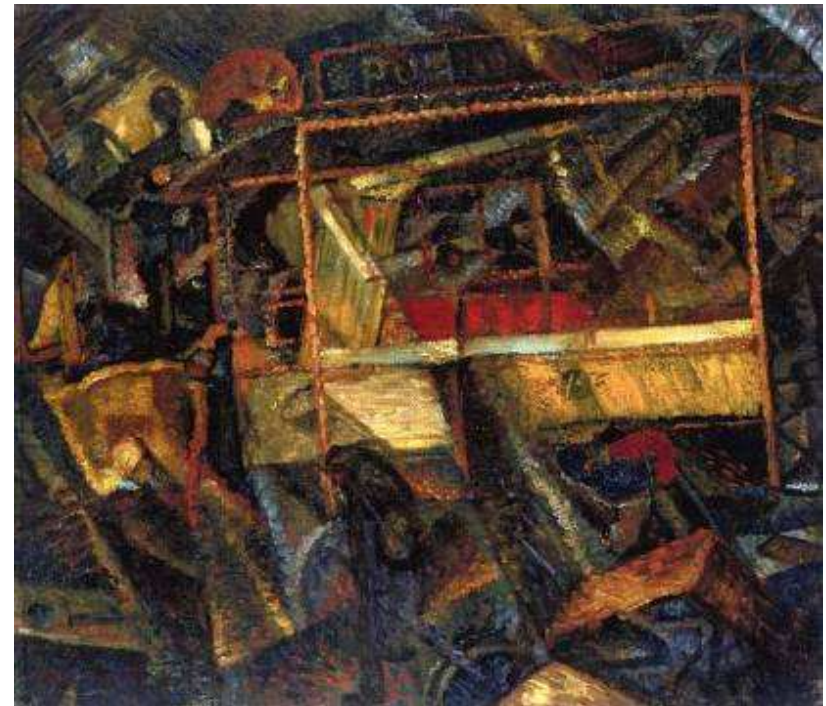

Carlo Carrà, Ce que m’a dit le Tramway, 1911

Huile sur toile, $52 \times 62 \mathrm{~cm}$,

Rovereto, Museo di Arte Moderna e Contemporanea di Trento e Rovereto 
Fig.14.

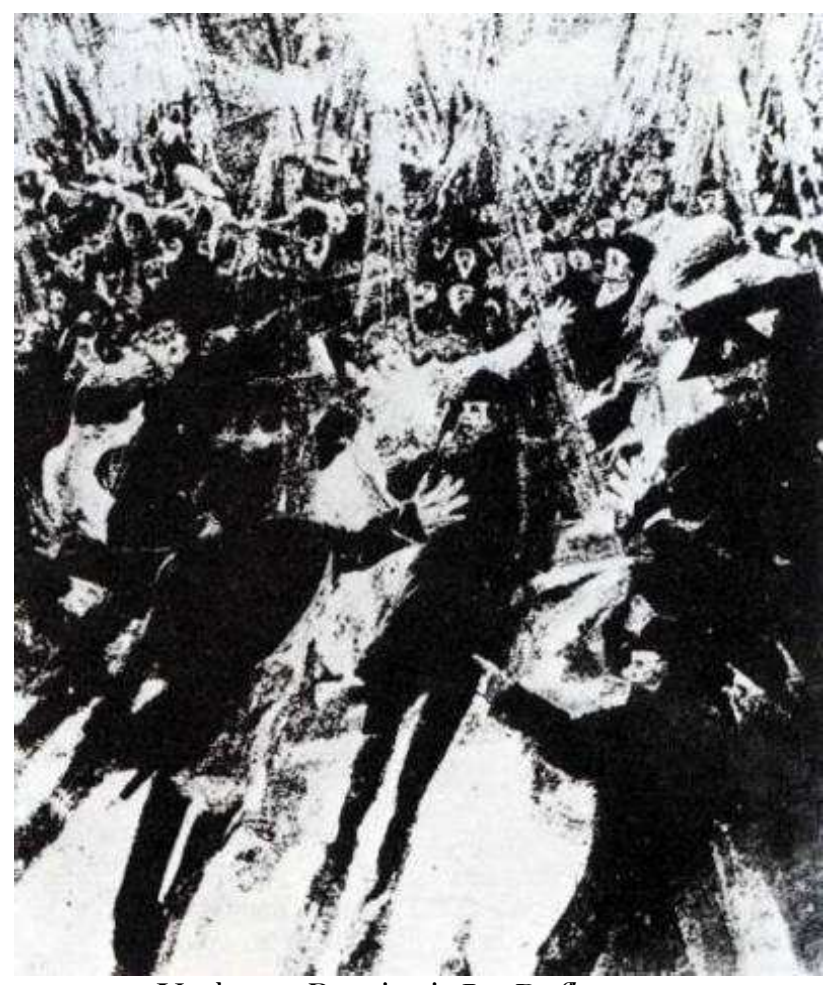

Umberto Boccioni, La Rafle, 1910

Huile sur toile, dimensions inconnues, Collection particulière

Fig.15.

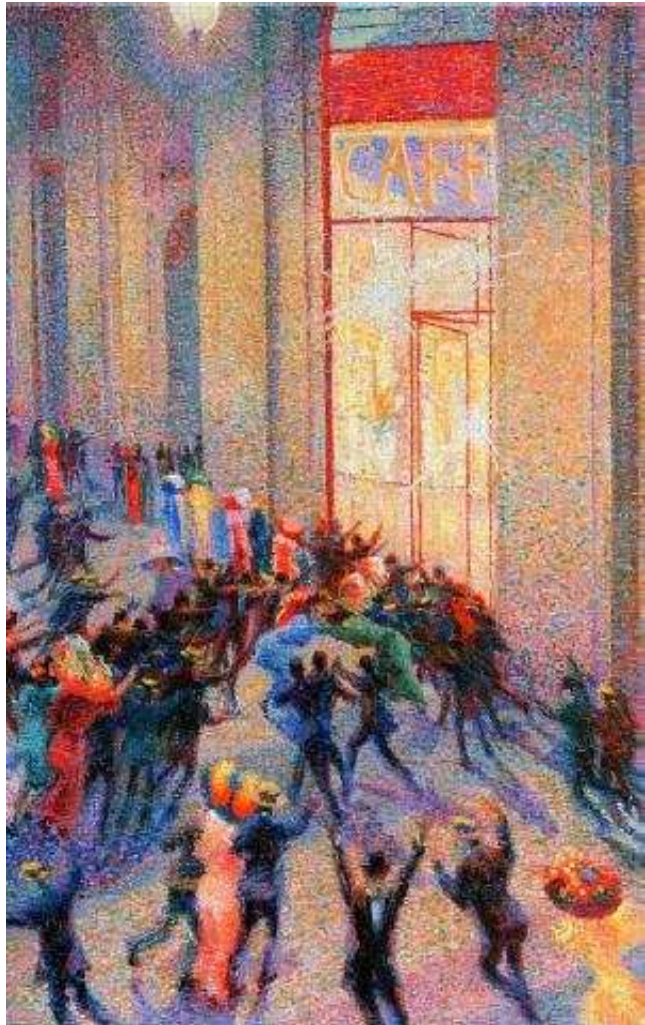

Umberto Boccioni, Echauffourée en Galerie, 1910

Huile sur toile, 76 x 64 cm, Milan, Pinacoteca di Brera 
Fig.16.

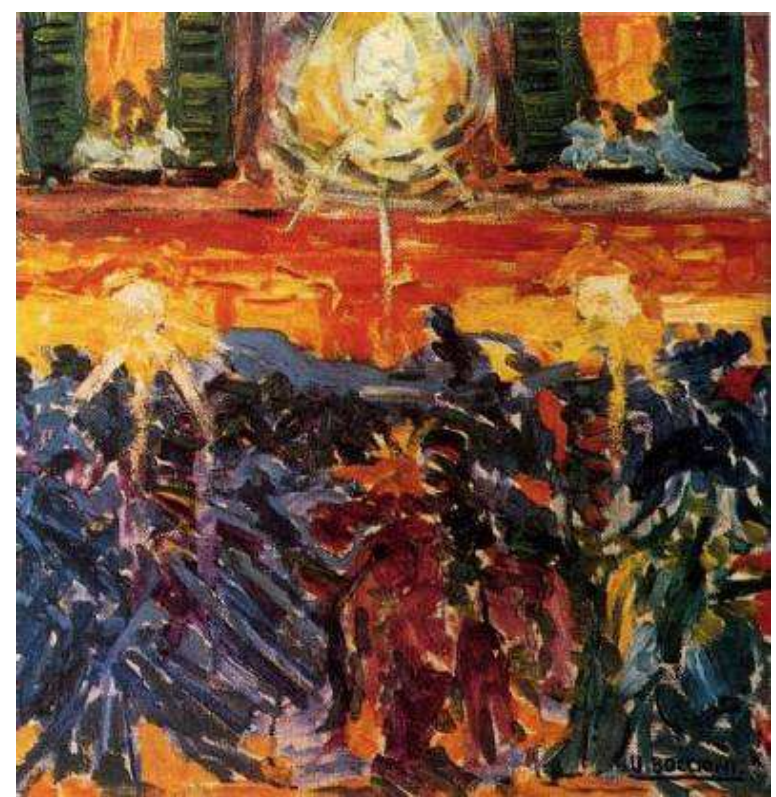

Umberto Boccioni, Echauffourée (Baruffa), c. 1911

Huile sur toile, 50,5 x 50,5cm, New York, The Museum of Modern Art

Fig.17.

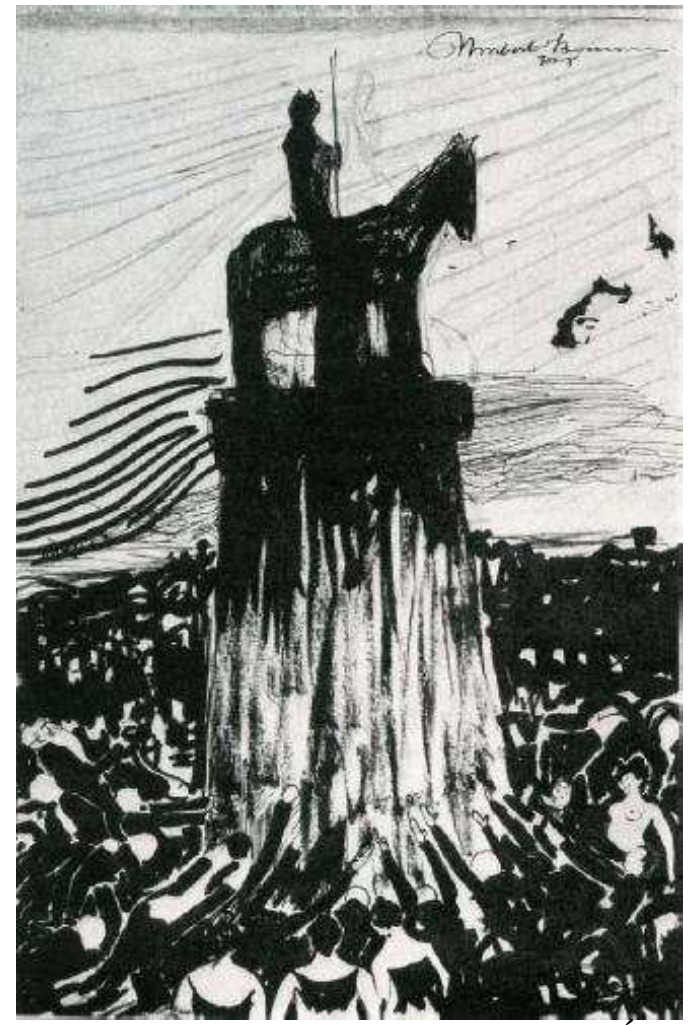

Umberto Boccioni, Foule autour d'un Monument Equestre, 1908

Encre sur papier, 35,9 x 24,2 cm, Birmingham, Winston Collection

Marinetti avait évoqué explicitement la foule comme thème de prédilection de l'avantgarde naissante. Dans le Manifeste du Futurisme, il proclamait : «nous chanterons les grandes foules agitées par le travail, le plaisir ou la révolte : les ressacs multicolores et polyphoniques 
des révolutions dans les capitales modernes $»^{56}$. Le travail, le plaisir, la révolte : les quatre toiles citées ci-dessus que Carrà, Boccioni, Severini et Russolo allaient peindre entre la fin 1910 et 1911 semblaient illustrer point par point la triade de Marinetti. En effet, les foules agitées par le travail habitaient La Ville qui monte dont titre originel était justement Le Travail; les foules agitées par le plaisir étaient emportées par les rythmes frénétiques de la danse pan-pan et les foules agitées par la révolte étaient les protagonistes des Funérailles de Galli l'Anarchiste et de La Révolte. Il est tout à fait probable que la valeur programmatique des quatre grandes toiles présentées à l'exposition Les Peintres Futuristes Italiens en février 1912 réside autant dans le thème représenté que dans le style de la représentation.

5.

Si on analyse la genèse de La Ville qui monte (fig.5) de Boccioni, on trouve une relation similaire entre la réflexion sur le thème de la foule et la recherche d'un nouveau langage pictural. Dans une lettre de 1910 adressée à « Ma chère (éternelle) mécontente » (peut-être s'agissait-il de sa maîtresse de longue date, Inès), il écrivait à propos du tableau qu'il était en train de retoucher :

Je comprends ainsi les paroles de Marinetti : aucun ouvrage qui n'ait un caractère agressif ne peut être un chef-d'œuvre. Et il me semble que le grand tableau le soit assez. Comme toujours, j'ai multiplié mon inspiration et le tableau est devenu plus peuplé et violent qu'auparavant. La foule a augmenté et j'espère donner à la plus petite figure la sensation d'allure fatale propre aux foules qui travaillent ${ }^{57}$.

L'expression allure fatale [andare fatale] résume très efficacement l'idée que les futuristes s'étaient faite de la foule. De nombreux passages de leurs écrits démontrent le vif intérêt qu'ils manifestèrent pour ce phénomène. Aux yeux de Boccioni, la foule se profile comme le pôle d'une attraction presque magnétique qui attire l'individu dans un «bouleversant tourbillon » ${ }^{58}$. À l'intérieur de la foule, le sujet dominé par des forces transcendant les limites de son individualité - et que, par conséquent, Boccioni appelle fatales -, sent sa volonté s'affaiblir et les bornes de sa conscience se dissoudre.

Cette interprétation de la foule présente des points communs très significatifs avec l'analyse des principes de l'esthétique futuriste conduite par Boccioni dans Dynamisme Plastique (1914). Si, en se mêlant à la foule, on peut se plonger dans le «tourbillon de la modernité $»^{59}$ et expérimenter une sorte de fusion panique avec la réalité, l'artiste futuriste, poussé par la conviction que sujet, objet et environnement forment une «unité indissoluble $»^{60}$, doit rejeter le principium individuationis, c'est-à-dire qu'il doit éliminer les contours clos et définitifs qui conduisent à une vision statique et fragmentaire du monde et proclamer le principe de la «compénétration des plans». Si le mouvement des foules communique une «sensation d'allure fatale », c'est à l'artiste de pressentir le devenir dynamique des objets et sa « loi de succession $»^{61}$, en révélant et matérialisant sur la toile les forces invisibles qui gouvernent la réalité. Comme on l'a vu, c'est précisément à ces principes que les solutions formelles mises au point par Carrà dans ses Funérailles répondaient: la même force centripète qui, ici, engloutit hommes et chevaux, entraîne et déforme les figures qui peuplent La Ville qui monte de Boccioni, tandis que Severini parvient à des résultats

\footnotetext{
${ }^{56}$ F. T. Marinetti, «Fondation et manifeste du futurisme (1909) », in G. Lista, Futurisme, op.cit., p. 87.

57 « A una signora (1911)», in U. Boccioni, Scritti Editi e Inediti, op.cit., p. 392.

${ }^{58}$ U. Boccioni, Dynamisme Plastique, op.cit., p. 88. Voir U. Boccioni, « Pittura scultura futuriste », op.cit., p. 175.

${ }^{59}$ Ibidem.

${ }^{60}$ Ibid., p. 86 [p. 120].

${ }^{61}$ Ibid., p. 80 [p. 118$]$.
} 
analogues en éparpillant les danseurs de pan-pan en milliers de fragments qui se recomposent sur la toile de façon casuelle et désordonnée.

Boccioni lui-même reconnaissait l'étroite relation qui existait entre la genèse de sa réflexion artistique et l'expérience de la foule. En effet, dans le chapitre VIII de Dynamisme Plastique, il faisait remonter certaines des plus importantes propositions du Manifeste Technique à des expériences éclairantes qu'il avait eues dans les rues de Milan :

Je me souviens que la célèbre affirmation Les seize personnes qui vous avez autour de vous dans un autobus etc.62 me vint à l'esprit au Corso [di Porta] Romana, en traversant une zone ensoleillée, qui débouchait de la Via Orti comme un torrent scintillant. Un autre énoncé : Que de fois sur la joue de la personne avec laquelle nous causons dans la rue nous voyons un cheval qui passe très loin, me vint à l'esprit en observant qu'un cocher qui passait au loin sur la Piazza del Duomo pénétrait dans la zone de lumière de la joue d'un prêtre arrêté devant la devanture des grands magasins Bocconi. Nos corps entrent dans les canapés sur lesquels nous nous asseyons et les canapés entrent en nous. L'autobus s'élance dans les maisons qu'il dépasse et, à leur tour, les maisons se précipitent sur l'autobus et se fondent avec lui : cette affirmation est à rattacher à des expériences sur des portraits et des études de la vie moderne que j’avais alors effectués après de très gros efforts dus à une analyse expérimentale presque mathématique. De même, l'idée Nous placerons le spectateur au centre du tableau m'est venue Piazza del Duomo, presque au coin de la Via Orefici, tandis que, sans bouger, j'observais l'effet provoqué par les gens qui avançaient, arrivaient à mon niveau et me dépassaient ${ }^{63}$.

Les coïncidences, les affinités, les renvois qui relient les représentations de la foule contenues dans les écrits de Boccioni, le sens qu'il attribuait à ses tableaux et la reconstruction qu'il suggérait de l'origine des principes de la peinture futuriste confirment l'hypothèse, formulée auparavant à propos de la genèse de Les Funérailles de l'Anarchiste Galli, selon laquelle la recherche formelle des futuristes (et pas seulement leur prédilection thématique) a été influencée par l'expérience de la foule en tant qu'agrégat social dans lequel s'établissent de nouveaux rapports entre individu et collectivité. Ce qui fascinait les peintres futuristes dans la foule - et dans la vie urbaine en général - n'était donc pas le dynamisme en lui-même ${ }^{64}$. Le culte de la vitesse dont le Manifeste de Marinetti représente l'expression la plus grandiloquente trouve peu de correspondances dans l'œuvre de Boccioni et de Carrà, où on ne trouve pas d'automobiles rugissantes. La nature profondément différente du dynamisme plastique "marqué par des thèmes explicitement cinétiques » propre à Balla, par rapport aux «composants idéologiques qui poussaient Boccioni à peindre un quartier avec des ouvriers au travail ou Carrà à s'intéresser à la gare de Milan » (fig.12) a été justement soulignée par Giovanni Lista ${ }^{65}$. La vitesse n'attirait pas Boccioni et Carrà ; mettre au point des expédients stylistiques pour traduire le mouvement sur la toile n'était pas leur but. Le dynamisme ne les intéressait pas, pourrait-on dire, en tant que manifestation du progrès technique. Un voyage en tram n'induisait pas les deux artistes à réfléchir sur les rythmes

\footnotetext{
${ }^{62}$ Boccioni se réfère à la phrase : «Les seize personnes que vous avez autour de vous dans un autobus en marche sont, tour à tour et à la fois, une, dix, quatre, trois ; elles sont immobiles et se déplacent ; elles vont, viennent, bondissent dans la rue, brusquement dévorées par le soleil, puis reviennent s'asseoir devant vous, comme des symboles persistants de la vibration universelle ». Voir « Manifeste des peintres futuristes », op.cit., p. 164 et «Pittura futurista », op.cit., p. 9.

${ }_{63}$ U. Boccioni, Dynamisme Plastique, op.cit., p. 67. Voir U. Boccioni, « Pittura scultura futuriste », op.cit., p. 141142.

${ }^{64}$ Selon une interprétation durable (et désormais stéréotypée) qu'on peut faire remonter à l'article de Roberto Longhi, «I pittori futuristi », La Voce 10 avril 1913, p. 1051, puis chez Id., Scritti Giovanili 1912-1922, vol. I, t. 1 de l'Edizione delle Opere Complete di Roberto Longhi, Florence, Sansoni, 1961, p. 47.

${ }^{65}$ G. Lista, «Gli anni dieci : il dinamismo plastico », in G. Lista et A. Masoero (dir.), Futurismo 1909-2009. Velocità+Arte+Azione, (catalogue de l'exposition, Milan, Palazzo Reale, 6 février-7 juin 2009), Milan, Skira, 2009, p. 100.
} 
frénétiques de la ville moderne : il aiguisait plutôt leur perception de la compénétration des individus les uns dans les autres et dans l'environnement.

Tom Gibbons a supposé que «la fusion cubiste des objets avec leur environnement [...] pourrait être entendue comme un moyen de peindre la croyance la plus importante de la tradition occulte idéaliste. La séparation des existences individuelles, qu'il s'agisse de celles des objets ou de celles des êtres vivants, est purement apparente et tous font, dans la réalité transcendantale, partie d'une unité interpénétrante et indivisible ${ }^{66}$. À la lumière de notre analyse, l'hypothèse d'une dépendance directe entre expérimentation futuriste et occultisme, proposée par Celant, nous semble encore moins convaincante. En effet, la représentation futuriste de l'invisible nous paraît plutôt enracinée tant dans des expériences sociales que dans des suggestions intellectuelles qui, interagissant, se renforcent mutuellement. Ainsi, Ringbom remarquait-il à propos des rapports entre Kandinsky et les doctrines théosophiques : «trouver de continuelles confirmations de ses propres convictions dans les écrits des autres semble être une double affaire : parfois cela permet non seulement d'accroître la foi en ses propres convictions, mais cela donne aussi plus de poids à certaines idées qui auparavant servaient seulement à étayer sa propre opinion $\gg^{67}$.

Il est probable que les futuristes aient trouvé dans l'expérience de la foule une plausible confirmation de leurs idées en matière de phénomènes médiumniques. Dans le même temps, il est possible aussi que l'occultisme ait contribué à orienter les futuristes vers une interprétation de la foule centrée sur la perception des forces invisibles qui dissoudraient les bornes de l'individualité et rendraient les corps perméables les uns aux autres. Mais encore, il se peut que les futuristes aient trouvé dans les sciences occultes une preuve supplémentaire de leur conception des dynamiques interindividuelles qui s'instauraient au sein des foules. L'existence supposée de forces de nature non identifiées mais bien réelles, invisibles à l'œil nu mais que le medium pouvait évoquer et diriger et qui pouvaient se condenser ou «s'extérioriser» sous forme d'auras phosphorescentes et d'ectoplasmes, finissait par légitimer l'intuition, jaillie de l'expérience de la foule, de forces arcanes qui dominaient les corps et l'intelligence des hommes.

Ce que nous avons dit vaut non seulement pour l'occultisme mais aussi pour d'autres facteurs qui, en interagissant avec les impulsions données par l'occultisme, intervinrent dans le processus de définition de la représentation futuriste de la foule.

6.

Au début du Xx ${ }^{\text {ème }}$ siècle, le thème de la foule s'imposa dans le contexte de l'anarchisme individualiste italien, dont l'hebdomadaire Il Grido della Folla, publié à Milan entre 1902 et 1907, fut l'expression la plus éloquente. Les anarchistes anti-organisateurs considéraient la foule comme la manifestation d'une socialité spontanée, non artificielle, non coercitive et qui ne limitait donc pas la liberté individuelle, et ils percevaient en elle une possible solution à la contradiction, propre à la pensée anarchiste, qui opposait la revendication de l'autonomie de

\footnotetext{
${ }^{66}$ "The Cubists" "fusion of objects with their surroundings" [...] may be seen as a method of depicting the most fundamental belief of the occult-idealist tradition: that the separateness of individual existences, whether of objects or of living creatures, is merely apparent, and that all are, in transcendental reality, part of one interpenetrating and indivisible unity», T. Gibbons, «Cubism and "the fourth dimension" in the context of the late nineteenth-century and early twentieth-century revival of occult idealism ", The Journal of the Warburg and the Courtauld Institutes 44, 1981, p. 130-147. La citation est à p. 143.

67 «To find repeated corroboration of one's convictions in the writings of other people-Ringbom a remarqué à propos des rapports entre Kandinsky et les doctrines théosophiques - may be a twofold affair; sometimes it not only increases one's faith in the convictions themselves, but also lends additional weight to the ideas that originally served as corroborations only » (S. Ringbom, « Art in the "Epoch of the Great Spiritual” », op.cit., p. 397).
} 
l'individu à la nécessité de parvenir à une unité supérieure des volontés et de préserver la dimension communautaire de la vie. Comme on l'a vu, on retrouve un intérêt similaire pour la dynamique des relations entre individu et collectivité dans la représentation iconographique que les futuristes donnèrent de la foule. Et cette analogie se révèle d'autant plus significative si l'on considère les rapports étroits que les futuristes (Carrà en particulier) entretinrent avec les milieux anarchistes et le groupe milanais du « Grido » ${ }^{68}$.

Du reste une telle représentation de la foule ne fut pas propre aux futuristes et aux anarchistes. Comme on le sait, le motif de la foule connut une fortune extraordinaire dans la culture européenne aux XIX ${ }^{\text {ème }}$ et XX ${ }^{\text {ème }}$ siècles. Il s'imposa dans les domaines de la littérature, de la politique et de la recherche sociale en relation avec la réflexion sur le déclin de l'individualisme libéral qui, avec la dénonciation de la «banqueroute de la science », fournit les principaux arguments au débat autour de la «crise de fin de siècle » ${ }^{69}$. Dans ce contexte, une nouvelle discipline naquit en France et en Italie, faisant de la foule son objet d'étude : la psychologie des foules.

En comparant les écrits de Scipio Sighele et de Gustave Le Bon ${ }^{70}$, les fondateurs de cette discipline, on s'aperçoit que, bien que différentes et, à certains égards, opposées, leurs interprétations convergeaient sur ce point: ils reconnaissaient tous deux les facultés spontanées d'organisation, tant puissantes que transitoires, des agrégats sociaux les plus hétérogènes. Des processus soudains d'assimilation se seraient amorcés au sein des foules transformant ainsi un fatras d'individus en une collectivité psychologiquement unitaire et cohérente, dotée d'une «âme » et d'une «intelligence ». Au moment où faisait irruption l'« âme de la foule » correspondait une annihilation de la volonté des individus : ces derniers se trouvaient transformés, comme écrivait Sighele, en des «automates qui se laissent entraîner $[\ldots]$ sans savoir et sans pouvoir réagir $\gg^{71}$.

L'analyse des psychologues présente d'évidentes analogies avec la représentation futuriste de la foule que nous avons examinée. Du reste, plusieurs indices nous font supposer que les futuristes avaient connu les théories des psychologues qui se diffuseraient bientôt ${ }^{72}$. Les explications de la psychologie et celles de l'occultisme se sont sans doute soutenues réciproquement. À cette époque, la distinction entre psychologie et occultisme n'était pas encore nettement démarquée et d'éminents psychiatres et médecins, parmi lesquels les italiens Enrico Morselli et Cesare Lombroso (dont Sighele fut l'élève) et les français Charles Richet et Camille Flammarion, consacrèrent leurs recherches aux phénomènes médiumniques $^{73}$. Il est vrai que des contrastes aigus se profilèrent entre, d'un côté, psychologues et psychiatres et, de l'autre, occultistes, et que l'intérêt de certains psychologues pour les phénomènes médiumniques contribua fortement à discréditer la parapsychologie et à l'éloigner définitivement de la psychiatrie ${ }^{74}$. Toutefois peut-être que "l'émergence à la fin du XIX ${ }^{e ̀ m e ~ s i e ̀ c l e ~ d e ~ n o u v e l l e s ~ t h e ́ o r i e s ~ t o u c h a n t ~ a ̀ ~ l a ~ n a t u r e ~ d e ~ l a ~ r e ́ a l i t e ́ ~}$ et à la nature de soi créa une ouverture vers les idées mystiques et occultes ». En effet, « la

\footnotetext{
${ }^{68}$ J'ai analysé la représentation anarchiste de la foule et reconstruit les rapports entre futuristes et anarchistes dans ma thèse de doctorat : C. Bertolotti, Anarchici e Futuristi. L'esperienza della folla, Università degli Studi di Siena, a.a. 2008-2009.

${ }^{69}$ Voir L. Mangoni, Una Crisi di Fine Secolo. La cultura italiana e la Francia fra Otto e Novecento, Turin, Einaudi, 1985 .

70 Voir S. Sighele, La Foule Criminelle. Essai de psychologie collective, Paris, Alcan, 1892 (éd. fr. revue et augmentée) et G. Le Bon, Psychologie des Foules, Paris, Alcan, 1895.

${ }^{71}$ S. Sighele, La Foule Criminelle, op.cit., p. 105.

${ }^{72}$ Sur les rapports entre Futurisme et psychologie des foules, voir C. Poggi, «Folla/Follia. Futurism and the crowd », Critical Inquiry 28, printemps 2002, p. 709-748. J'ai discuté les hypothèses de Poggi dans ma thèse de doctorat : C. Bertolotti, Anarchici e Futuristi, op.cit..

73 Voir C. Lombroso, Ricerche sui Fenomeni Ipnotici e Spiritici, Turin, Utet, 1909 ; E. Morselli, Psicologia e Spiritismo. Impressioni e note critiche sui fenomeni medianici di Eusapia Paladino, 2 vols., Turin, Bocca, 1908.

${ }_{74}$ Voir H. Wolffram, «Parapsychology on the couch: the psychology of occult belief in Germany, c. 1870$1939 »$, Journal of the History of Behavioral Sciences 42(3), été 2006, p. 237-260.
} 
psychologie et les intérêts mystiques ou occultes étaient vus, au cours de la période symboliste, comme des chemins complémentaires permettant d'explorer la réalité intérieure de soi et du monde $\gg^{75}$. De nombreuses études explorant la fascination que l'occultisme et le mysticisme exercèrent sur les plus importants représentants des avant-gardes artistiques européennes révèlent les multiples connexions qui existent entre psychologie et sciences occultes $^{76}$ : le cas du futurisme que nous avons examiné semble en fournir une épreuve ultérieure.

Boccioni connaissait sans doute les études de Richet. En 1914, il écrivait : "Notre audace futuriste a déjà forcé les portes d'un monde inconnu. Nous cherchons à créer quelque chose d'analogue à ce que le physiologiste Richet appelle hétéroplastie ou idéoplastie. Le mystère biologique de la matérialisation médiumnique est pour nous une certitude, une clarté dans l'intuition du transcentalisme physique et des états d'âme plastiques » ${ }^{77}$. Quant à Flammarion, on peut bien supposer que Boccioni avait lu son oeuvre concernant les phénomènes médiumniques, traduite en italien au début du siècle par le mari de sa sœur Amelia, le professeur Guido Valeriano Callegari, qui en 1910 avait également écrit une courte biographie de l'astronome français ${ }^{78}$.

Si les futuristes trouvaient dans la psychologie et l'occultisme des confirmations de l'existence de forces invisibles et pourtant réelles, les progrès de la science et de la technique ne contribuèrent pas moins efficacement à consolider leur certitude. En effet, Boccioni soulignait :

Cette matérialisation du fluide, de l'éthéré, de l'impondérable; cette transposition dans le concret de ce qu'on pourrait appeler le nouvel infini biologique et que la fièvre de l'intuition illumine, c'est peut-être de la littérature? Toutes les recherches humaines de notre époque n'aspirentelles pas sans doute à cet impondérable qui est en nous, autour de nous et pour nous ?79

La théorie électrique de la matière, « selon laquelle la matière ne serait que de l'énergie, de l'électricité condensée et n'existerait que comme force », aussi bien que «les dernières hypothèses scientifiques, les innombrables possibilités que nous offrent la chimie, la physique, la biologie et toutes les découvertes de la science, la vie de l'infiniment petit» contribuaient, selon lui, à intensifier son intuition et l'entraînaient à rechercher « dans la sensibilité plastique des analogies avec ces nouvelles et merveilleuses conceptions naturelles » :

Nous pouvons affirmer et créer plastiquement les vibrations, les émanations, les densités, les émeutes, le halo invisible entre l'objet et son action, la synthèse analogique vivant aux confins entre l'objet réel et sa plastique idéale, bref, tout ce que représente la vie de l’objet ${ }^{80}$.

\footnotetext{
75 « The emergence in the later nineteenth century of new teories about the nature of reality and the nature of the self created an openness towards mystical and occult ideas. [...] Psychology and mystical or occult pursuits were seen in the Symbolist era as complementary routes for exploring the inner reality of self and world» (L. Dalrymple Henderson, «Editor's statement: Mysticism and occultism in modern art », Art Journal 46(1), printemps 1987, p. 5-6).

${ }^{76}$ Je me réfère aux études recueillies dans le numéro monographique de Art Journal, (Mysticism and Occultism in Modern Art), op.cit..

77 U.B occioni, «Pittura scultura futuriste», op.cit., p. 201. Le terme «idéoplastie » a été utilisé par Charles Richet à l'occasion des expériences conduites avec les mediums Linda Gazzera et Eva Carrière entre 1912 et 1914. Ces expériences auraient démontré, selon Richet, l'existence de matérialisations de figures humaines, qui étaient la reproduction objectivée de portraits et de dessins tombés sous les yeux des médiums. Voir C. Richet, Traité de Métapsychique, Paris, Alcan, 1922, passim. Nous ne savons pas pourtant quels écrits de Richet, précédents le Traité, Boccioni eut la possibilité de lire.

78 Voir C. Flammarion, Le Forze Naturali Sconosciute, Milan, Sonzogno, s.d, (début XXème siècle) [éd. or. C. Flammarion, Les Forces Naturelles Inconnues, 1865] et G. V. Callegari, Flammarion, Florence, La rinascita del libro di A. Quattrini, 1910. À propos des rapports de Boccioni avec son beau-frère voir V. Baradel, Gli Anni Veronesi di Amelia Boccioni, in Boccioni a Verona, San Pietro in Cariano, Edizioni della Vita Nova, 2010, p. $27-34$.

${ }^{79}$ U. Boccioni, Dynamisme Plastique, op.cit., p. 104. Voir U. Boccioni, « Pittura scultura futuriste », op.cit., p. 201.

80 Ibid., p. 105 [p. 201].
} 
L'intérêt des artistes pour les phénomènes psychiques et les sciences occultes a été généralement interprété « comme un antidote au climat scientifique empiriste et étroitement positiviste qui prévalait au début du siècle ${ }^{81}$. L'adhésion de Kandinsky aux doctrines théosophiques impliquait, en effet, une prise de conscience de la faillite de la science positiviste $^{82}$. Au contraire, Boccioni considérait de la même manière les phénomènes médiumniques et les découvertes scientifiques et les utilisaient toutes les deux, en les associant, pour confirmer ses propres intuitions :

Autour de nous passent des énergies qu'on observe et étudie; de nos corps émanent des fluides de puissance, d'attraction ou de répulsion $[\ldots]$; on prévoit la mort de quelqu'un à des centaines de kilomètres à la ronde; les pressentiments nous insufflent de la force ou nous écrasent de terreur. Les ondes hertziennes emportent à des milliers de kilomètres, au-delà des océans, à travers les déserts, les pulsions fébriles des races. Le microbe est poursuivi dans les profondeurs insondables de la matière, on l'étudie dans ses habitudes, on le photographie et on le fixe dans son individualité infinitésimale. Les électrons tournoient dans l'atome par dizaines de milliers, séparés les uns des autres comme les planètes du système solaire et possédant comme elles une orbite et une vitesse inconcevables pour nous, et l'atome est déjà invisible [sic] à nos yeux et à nos instruments optiques... ${ }^{83}$.

Paroles qui font écho de façon étonnante aux lignes écrites en 1910 par son beau-frère Callegari à propos de Flammarion :

Selon lui - cette théorie a pris aujourd'hui le dessus - la matière n'existe pas comme nos sens l'aperçoivent, qui nous donnent seulement les impressions incomplètes d'une réalité inconnue; l'analyse nous démontre que la matière est une forme d'énergie et qu'elle serait constituée, comme la lumière, la chaleur, l'électricité, d'une espèce de mouvement [...]. La matière n'est qu'une façon de mouvement, une expression de la force, l'atome même a disparu et est devenu des tourbillons insaisissables (électrons) : l'univers est dynamismes.

Une même «attention pour les mondes invisibles » ${ }^{85}$ animait savants, psychologues et occultistes.

7.

Dans un article paru le 10 juin 1902 dans Il Grido della Folla, l'hebdomadaire anarchiste publié à Milan que nous avons mentionné plus haut, Ettore Molinari, qui en avait été le fondateur, écrivait :

Observez par exemple les milliers de personnes qui marchent et se croisent sur le trottoir d'une rue fréquentée dans une grande ville : aucune loi écrite ne règle ce mouvemente fébrile et continu de milliers de personnes. L'usage et le bon sens les guident à droite ou à gauche; des hommes, des femmes, des enfants se croisent dans toutes les directions. Personne ne se heurte à un autre, personne ne se dispute le passage avec quelqu'un d'autre. Donc, le mouvement dans les rues et les établissements publics (l'une des

\footnotetext{
${ }^{81}$ M. Braun, « Fantasmes des vivants et des morts », op.cit., p. 16.

82 Voir S. Ringbom, «Art in "the Epoch of the Great Spiritual” », op.cit., p. 386, 389, 395.

${ }^{83}$ U. Boccioni, Dynamisme Plastique, op.cit., p. 105-106. Voir U. Boccioni, « Pittura scultura futuriste », op.cit., p. 201.

${ }^{84}$ G. V. Callegari, Flammarion,op.cit., p. 51. L’italique est dans le texte original.

${ }^{85}$ M. Braun, « Fantasmes des vivants et des morts », op.cit., p. 16.
} 
manifestations les plus importantes de la vie humaine) est typiquement anarchiste, c'est-à-dire qu'il se produit sans lois écrites ${ }^{86}$.

Friedrich Engles avait jugé «l'accord tacite ${ }^{87}$ qui disciplinait la foule de Londres comme la dégoutante expression de l'individualisme et de l'égoïsme propres à la société bourgeoise : l'anarchiste Molinari, renversant ce jugement, valorisait avec enthousiasme la puissance qu'avait la foule de s'organiser spontanément. Dans le mouvement de la foule, Molinari et ses amis reconnaissaient le reflet de l'«harmonie universelle» qui, comme Kropotkine l'enseignait, gouvernait aussi bien la société que la nature. En effet, afin d'étayer une interprétation de la foule comme forme de socialité naturelle, les anarchistes comme les futuristes faisaient allusion à des langages et images tirés des sciences. On ne sera pas surpris de découvrir maintenant qu'au début du XXème siècle Molinari était l'un des plus illustres chimistes italiens ${ }^{88}$.

$\mathrm{Si}$ on peut parler de naturalisme, il est évident qu'il s'agit ici de naturalisme mystique. En effet, pour confirmer leur interprétation de la foule comme forme sociale naturelle, les anarchistes, de la même façon que les futuristes, étaient contraints de supposer que la syntonie, qui s'instaurait dans la rue parmi des individus qui ne se connaissaient pas, était le fruit des forces arcanes qui les dirigeaient. Beaucoup plus tard, Luigi Galleani indiqua encore dans la foule le sujet révolutionnaire sur lequel les anarchistes auraient pu compter : "Cette masse qui échappe aux cadres de parti, écrivait-il, est la force occulte, mais sûre, de notre mouvement $»^{89}$.

Costanza Bertolotti est docteur en Anthropologie, Histoire et Théorie de la Culture (Université de Sienne - Istituto italiano di scienze umane). Elle a mené une recherche sur les rapports entre culture anarchique, futurisme et représentation de la foule au début du XX⿳亠丷⿵冂丶 Arts de l'EHESS avec un projet de recherche concernant la dialectique entre refus et survivance de la tradition dans le futurisme.

\footnotetext{
86 «Perché siamo socialisti anarchici? IV », Il Grido della Folla I(8), 10 juin 1902, p. 2.

87 «Übereinkunft zwischen ihnen die stillschweigende», F. Engels, Die Lage der Arabeitenden Klasse in England nach eigener Anschauung und authentischen Quellen, Stuttgart, J.H.W. Dietz, 1892, p. 23.

88 À propos d'Ettore Molinari, des rapports entre les représentations de la foule (anarchistes et futuristes) et le paradigme scientifique en vogue entre la fin du XIX ${ }^{\text {ème }}$ siècle et le début du XX ${ }^{\text {ème }}$ voir C. Bertolotti, Anarchici e Futuristi, op.cit.. Il s'agit d'une recherche en cours dont je me limite à faire ici une allusion très rapide.

${ }^{89}$ Minin [Luigi Galleani], Attenti ai mali passi!, cité par M. Antonioli, « La nascita dell'UAI e gli anarchici individualisti e antiorganizzatori », in L'Unione Anarchica Italiana. Tra rivoluzione europea e reazione Fascista (1919-1926), Milan, Zeroincondotta, 2006, p. 104.
} 